\title{
Fish Lateral Line Inspired Flow Sensors and Flow-aided Control: A Review
}

\author{
Yufan Zhai ${ }^{1}$, Xingwen Zheng ${ }^{{ }^{*}}$, Guangming Xie $^{1,2,3^{*}}$ \\ 1. State Key Laboratory for Turbulence and Complex Systems, Intelligent Biomimetic Design Lab, College of Engineering, \\ Peking University, Beijing 100871, China \\ 2. Institute of Ocean Research, Peking University, Beijing 100871, China \\ 3. Peng Cheng Laboratory, Shenzhen 518055, China
}

\begin{abstract}
Any phenomenon in nature is potential to be an inspiration for us to propose new ideas. Lateral line is a typical example which has attracted more interest in recent years. With the aid of lateral line, fish is capable of acquiring fluid information around, which is of great significance for them to survive, communicate and hunt underwater. In this paper, we briefly introduce the morphology and mechanism of the lateral line first. Then we focus on the development of artificial lateral line which typically consists of an array of sensors and can be installed on underwater robots. A series of sensors inspired by the lateral line with different sensing principles have been summarized. And then the applications of artificial lateral line systems in hydrodynamic environment sensing and vortices detection, dipole oscillation source detection, and autonomous control of underwater robots have been reviewed. In addition, the existing problems and future foci in this field have been further discussed in detail. The current works and future foci have demonstrated that artificial lateral line has great potentials of applications and contributes to the development of underwater robots.
\end{abstract}

Keywords: lateral line, artificial lateral line, bio-inspired flow sensor, flow-aided control, underwater robot Copyright $(C)$ The author(s) 2021.

\section{Introduction}

Comparing with extracting land resources, it's more difficult to exploit marine resources. Due to the complexity of underwater environment, the causticity of the seawater, the high pressure and the poor visibility in the sea, and the strong interference to the sensors, people are facing extremely harsh conditions when exploiting marine resources. With the rapid development of machine manufacturing and artificial intelligence, robots serve significant functions in resource exploitation.

However, the development of underwater robots is more difficult than that of land robots because of the above-mentioned reasons. With the further exploration of marine resources, the disadvantages of traditional underwater robots are gradually highlighted: large size, complex system, low control flexibility, high energy consumption, low efficiency, and lack of autonomy.

Biomimetics which acts as a new comprehensive subject provides another way for underwater robot manufacturing. Many researchers have taken inspiration from aquatic organisms and developed new underwater robots from the bionic perspective, commonly known as biomimetic underwater robots. For aquatic organisms, they have lived in marine environment for billions of years and adapted to the environment through evolution. They have the advantages of high maneuverability, high sensitivity to changes in surrounding environment and high energy efficiency, etc. Inspired by the excellent performance mentioned above, various kinds of biomimetic underwater robots have been developed successfully: robotic fish $^{[1-4]}$, robotic dolphin ${ }^{[5]}$, robotic snake ${ }^{[6]}$, robotic turtles ${ }^{[7]}$, robotic salamanders ${ }^{[8]}$, etc.

Owing to the complexity of underwater environment, robots must be equipped with well-developed underwater sensing systems, which can help them to adapt to the environment and perform underwater tasks. Due to the particularity of water environment, land sensors cannot be directly used under the water, which restricts the development of sensing technology for underwater robots to some extent. To solve this problem, scientists have taken inspiration from marine life once again. Over billions of years of evolution in the ocean, fish have developed advanced systems for sensing the

\footnotetext{
*Corresponding author: Xingwen Zheng, Guangming Xie E-mail: zhengxingwen@pku.edu.cn, xiegming@pku.edu.cn
} 
water environment. Lateral line is such a unique sensing system of fish and plays a key role for fish behaviours in complex water environment. The research and development of Artificial Lateral Line (ALL) systems referring to an array of sensors installed on the underwater vehicle will greatly improve the perception ability of the robots, which is advantageous for underwater missions.

In this paper, we firstly describe the biology of lateral line and the models which have been established to illustrate the mechanisms in section $\mathbf{2}$. In section $\mathbf{3}$, we review the sensors that are inspired by the lateral line and based on different sensing principles according to the categories. In section 4, we present applications of ALL in hydrodynamic environment sensing and vortices detection. In section 5, we introduce results in dipole oscillation source detection with the help of ALL using different methods. Finally, we discuss the flow-aided control of underwater robots using ALL systems for different purposes. At the end of the article, we discuss the problems of current studies and try to put forward possible improvement strategies for further studies.

\section{Mechanisms and models of the fish lateral line}

Lateral line is the most highly differentiated structure in the sensory organs of the skin, which is typically sulcus or tubular. It is a sensory organ peculiar to fish and aquatic amphibians and important for them to maneuver in the darkness ${ }^{[9]}$. The sensory unit of the lateral line is the neuromast, a receptor that consists of sensory hair cells and support cells ${ }^{[10]}$. There are two types of lateral line neuromasts: Superficial Neuromast (SN) and Canal Neuromast $(\mathrm{CN})$, as shown in Fig. 1. Both of these two kinds of neuromasts can sense the stimulation generated by water flow through sensory cells. Due to the difference in distribution, number and morphology of sensory cells, the two kinds of neuromasts have different functions ${ }^{[11]}$.

The SNs which act as displacement sensors are free-standing on the skin or on pedestals grown above the skin. They are usually located in lines on the fish body ${ }^{[13]}$. The ability of sensing the flow direction and velocity is mainly realized by the SNs which are sensitive to the displacement and respond to the low-frequency direct current component. When the water flow and the fish surface move relatively to each other, the SNs bend, causing the neuromasts below to produce nerve impulses which will be transmitted from nerve endings to the nerve centers of the brain. Under such a mechanism, fish can sense the flow information with the help of SNs. On the other hand, the CNs are equivalent to pressure gradient sensors which can sense pressure gradient and are sensitive to acceleration and respond to high-frequency components. The $\mathrm{CNs}$ are located in the lateral line canals that are full of mucus under the epidermis of fish and communicate with the external water environment through some small holes ${ }^{[14]}$. When there is a velocity gradient between adjacent holes, the pressure difference will be generated, leading to the fluid movement in the lateral line canals, which triggers the nerve impulse. The lateral line system composed of $\mathrm{CNs}$ and SNs can sense various stimuli from different directions, so that fish can acquire enough information for a full sense of surrounding water environment.

Scientists have established theoretical models to describe the sensing mechanisms of these neuromasts and to study the interactions between flow and fish, which is instructive to the development of ALL systems. As shown in Fig. 2, the $\mathrm{CN}$ can be regarded as a rigid hemisphere cupula sliding over a frictionless plate in biomechanics which is coupled with a linear spring ${ }^{[15,16]}$. For the forces on the $\mathrm{CNs}$, the governing equation can be described as:

$$
F_{m}+F_{k}=F_{u}+F_{b} .
$$

The terms on the left are the inertial force and the structural stiffness resistance which can be written as the following forms respectively:

$$
\begin{gathered}
F_{m}=M \frac{\mathrm{d}^{2} Y(t)}{\mathrm{d} t^{2}}, \\
F_{k}=K Y(t),
\end{gathered}
$$

where $M$ represents the cupula mass. $Y(t)$ represents the displacement induced by the passing flow. $K$ is the sliding stiffness. The terms on the right are the hydrodynamic drag force and the buoyant force due to the pressure difference which can be defined as the following forms: 


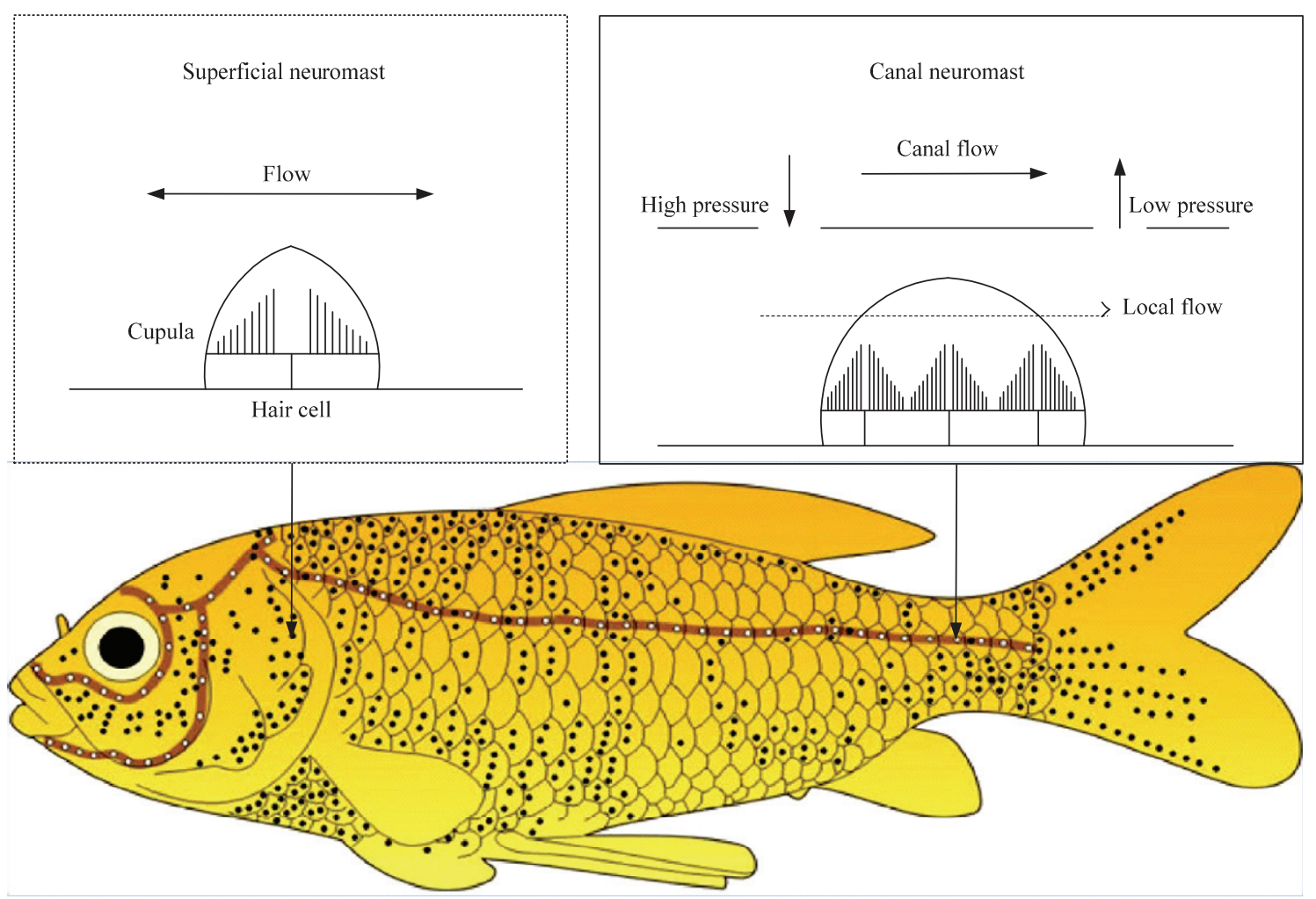

Fig. 1 Lateral line and neuromasts of a fish. Black dots represent locations of SNs, and white dots show the approximate locations of canal pores $^{[12]}$.
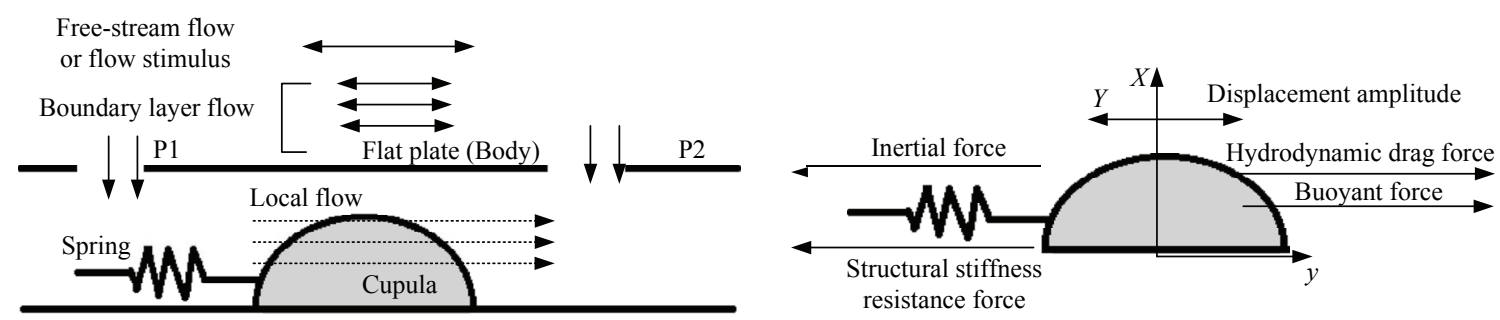

Fig. 2 Biomechanical model and force analysis for a $\mathrm{CN}^{[15]}$.

$$
\begin{aligned}
& F_{u}=D\left[\frac{\mathrm{d} Y(t)}{\mathrm{d} t}-\frac{\mathrm{d} W(t)}{\mathrm{d} t}\right] \\
& F_{b}=M \frac{\mathrm{d}^{2} W(t)}{\mathrm{d} t^{2}}
\end{aligned}
$$

$M \frac{\mathrm{d}^{2} Y(t)}{\mathrm{d} t^{2}}+K Y(t)=D\left[\frac{\mathrm{d} Y(t)}{\mathrm{d} t}-\frac{\mathrm{d} W(t)}{\mathrm{d} t}\right]+M \frac{\mathrm{d}^{2} W(t)}{\mathrm{d} t^{2}}$

where $D$ is the drag coefficient and $W(t)$ is the displacement of external flow. Thus $\frac{\mathrm{d} Y(t)}{\mathrm{d} t}-\frac{\mathrm{d} W(t)}{\mathrm{d} t}$ represents the relative velocity of the cupula and external flow. According to the above results, the governing equation can be written as:

In order to describe the sensitivity of CNs, considering that the equation is linear, the displacement can be decomposed into different frequencies. At a certain frequency, $Y(t)$ and $W(t)$ are the steady-state oscillatory displacement and the oscillating flow displacement with a form of $Y(t)=Y_{0}(f) e^{i 2 \pi f t}$ and $W(t)=W_{0}(f) e^{i 2 \pi f t}$. So the velocity of the flow is expressed as: 


$$
V(t)=\frac{\mathrm{d} W(t)}{\mathrm{d} t}=i 2 \pi f W_{0}(f) e^{i 2 \pi f t}=V_{0}(f) e^{i 2 \pi f t},
$$

The frequency-dependent sensitivity of the CNs is defined as the ratio of the displace amplitude of cupula and the velocity amplitude of flow, which is shown in the following formula:

$$
\begin{aligned}
S_{C N}(f) & =\frac{Y_{0}(f)}{V_{0}(f)} \\
=\frac{1}{2 \pi f_{t}} & \frac{1+\frac{\sqrt{2}}{2}(1+i) \sqrt{\frac{f}{f_{t}}}+\frac{1}{3} i \frac{f}{f_{t}}}{N_{r}+i \frac{f}{f_{t}}-\frac{\sqrt{2}}{2}(1-i)\left(\frac{f}{f_{t}}\right)^{\frac{3}{2}}-\frac{1}{3}\left(\frac{f}{f_{t}}\right)^{2}},
\end{aligned}
$$

where $f_{t}$ is the transition frequency expressed as $f_{t}=\frac{\mu}{2 \pi \rho_{w} a^{2}}$ which determine the viscous $\left(f<f_{t}\right)$ or the inertial $\left(f>f_{t}\right)$ force dominates the fluid forces applied on the cupula. $\mu$ is the dynamic viscosity of fluid. $N_{r}=\frac{K a \rho_{w}}{6 \pi \mu^{2}}$ is the resonance factor which represents the resonance properties.

Different from the $\mathrm{CN}$, the $\mathrm{SN}$ is modeled as two connecting beams with different bending rigidity, as shown in Fig. 3. The distal beam is more flexible than the proximal beam because of the difference in material properties of the proximal and distal parts. A spring is used to simulate the torsional stiffness generated by the hair beam. The governing equation is different from that

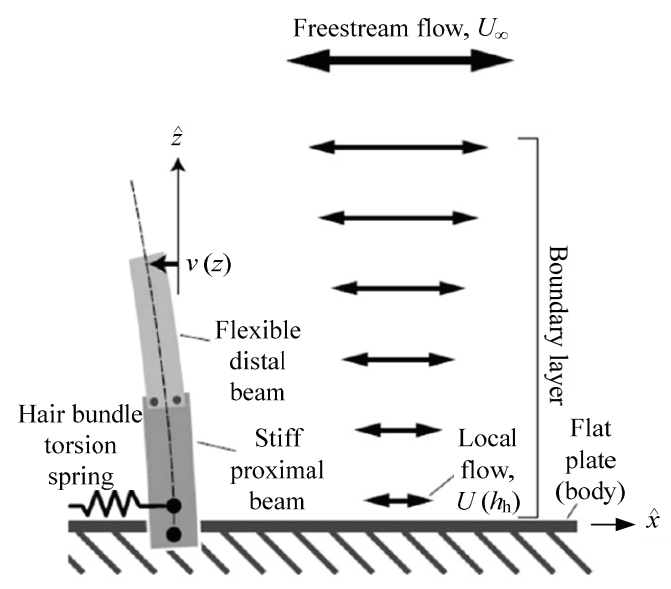

Fig. 3 Biomechanical model of a $\mathrm{SN}^{[17]}$. of CNs, forces applied on the beams are also functions of elevation $z$ due to the uneven distribution of forces when a beam bends. The equation is expressed as:

$$
F_{m}(z)+F_{e}(z)=F_{u}(z)+F_{a}(z)+F_{b}(z) .
$$

The terms from left to right represent the inertial force, the elastic stiffness term, the hydrodynamic drag force, the acceleration reaction force and the buoyant force respectively. Similar to CNs, the sensitivity of SNs is defined as the ratio of cupula deflection $v(H)$ at the height of the beam and the free-stream velocity $U_{\infty}$, which is written as:

$$
\begin{aligned}
S_{S N}(f)= & \frac{v(H)}{U_{\infty}} \\
= & -\frac{i b_{w}}{2 \pi f b_{m}}\left[1-\frac{i \pi f b_{m} \delta^{4}}{2 E I+i \pi f b_{m} \delta^{4}} e^{-\frac{H(1+i)}{\delta}}\right] \\
& +\sum_{j=0}^{3} C_{j} e^{i^{j} H} \sqrt[4]{\frac{2 \pi f b_{m}}{E I}},
\end{aligned}
$$

where $C_{j}$ represents a sequence of four integration constants. $H$ is the height of the top of the beam. $E I$ is the bending modulus of the beam. $\delta$ is the thickness of boundary layer expressed as $\delta=\sqrt{\frac{2 \mu}{\rho_{w} \omega}}$, where $\omega$ is the angular speed of the stimulus. More details on the parameters $b_{m}$ and $b_{w}$ can be referred to Ref. [17].

Fig. 4 shows the propagation paths of lateral line neuromasts. On one hand, for CNs, the propagation path is divided into two steps. At the first step, the velocity or acceleration of the external free flow is converted into the velocity of the local flow with the help of the boundary layer and canals. Flow outside induces the pressure difference between the canals, which triggers flow velocity inside the canals. At the second step, canal flow applies fluid forces on the cupula and results in the $\mathrm{CN}$ deflection. On the other hand, for SNs, at the first step, the velocity or acceleration of the external free flow is converted into the velocity of the local flow similarly without the reflection of canal flow. At the second step, SNs deflects under the forces induced by local flow ${ }^{[15]}$.

With the assistance of SNs and CNs, fish can detect the flow direction and speed and pressure gradients respectively. Moreover, SNs can distinguish fields in a 


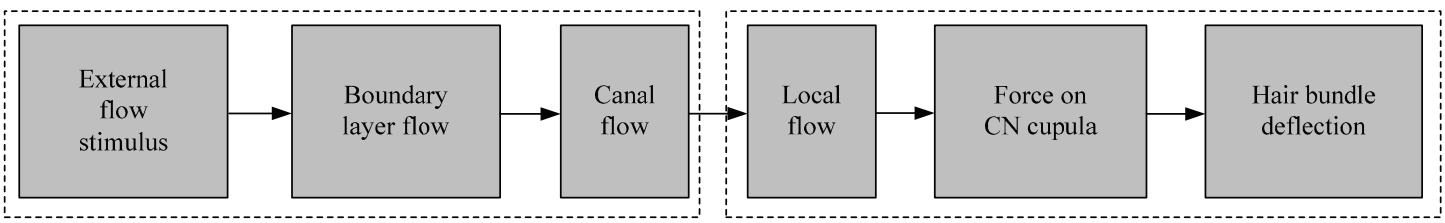

(a)

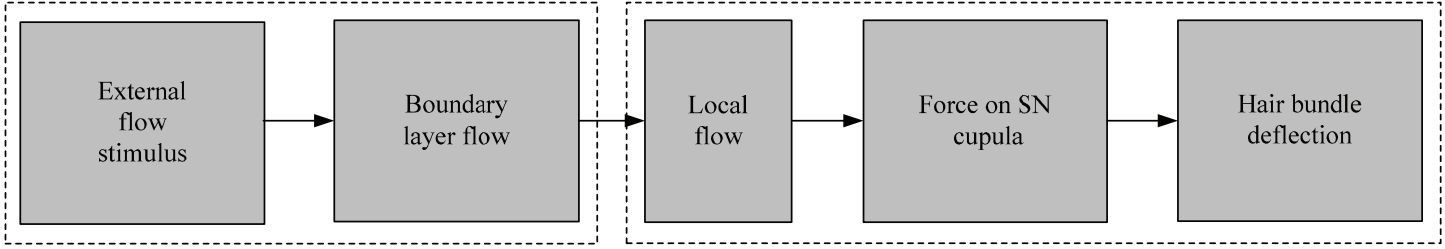

(b)

Fig. 4 Propagation paths of lateral line neuromasts. (a) Propagation path of CNs; (b) propagation path of SNs ${ }^{[15]}$.

spatial uniform flow and in a turbulent flow, while CNs only respond to a non-uniform flow field, such as the fluctuation of water produced by a vibrating sphere or a swimming fish.

\section{The existing ALL sensors and systems}

\subsection{ALL sensor unit}

As mentioned above, scientists have established mathematical models to interpret the mechanisms that how fish acquire fluid information assisted by lateral line. The results can be an inspiration of ALL. Owing to the limitations of existing technologies for underwater detection such as scattering and multipath propagation issues for acoustic sensors and turbidity of the sea for optical sensors $^{[15]}$, varieties of fish lateral line inspired sensors were developed based on different sensing mechanisms, including piezoresistive, piezoelectric, capacitive, optical, thermal and magnetic effect. ALL sensors can be used alone to detect the flow velocity and pressure distribution where other methods lose efficacy. Moreover, we can use moving robotic fish boarded with ALL sensors for a better perception of the surrounding environment. The research status of ALL sensors with different sensing mechanisms will be discussed below.

\subsubsection{Piezoresistive ALL sensors}

Piezoresistive sensor is a device based on the piezoresistive effect of the semiconductor material on the substrate. Piezoresistive effect refers to a phenomenon that the electrical resistance of the material changes while it is subjected to force. As a result, the bridge on the substrate produces corresponding unbalanced output. In this way, the substrate can be directly used as an element to measure pressure, tension etc. And then based on the quantity measured directly, the information about the environment is available. Fig. 5 shows various piezoresistive ALL sensors mentioned below.

In 2002, Fan et al. firstly made a major breakthrough in piezoresistive ALL sensors fabrication using combined bulk micromachining methods and an efficient three-dimensional assembly method named Plastic Deformation Magnetic Assembly (PDMA) process. They leveraged PDMA to realize the vertical cilium, which was important in the hair cell. A single sensor was composed of an in-plane fixed-free cantilever mainly made of Boron ion diffused Si using etching technology, a vertical artificial cilium attached at the free end and a strain gauge located at the base of the horizontal cantilever which was used to sense the bending of the vertical cilium. Subjected to the impact of local flow, the vertical cilium bent and transferred the influence to the cantilever beam. The corresponding results were measured by the strain gauge. The sensors were used to detect laminar flows ranging from $0.1 \mathrm{~m} \cdot \mathrm{s}^{-1}$ to $1 \mathrm{~m} \cdot \mathrm{s}^{-1[18]}$.

In 2003, Chen et al. compared the above sensor with the hot-wire anemometer and improved the design by rigidly connecting the vertical hair to the substrate and placing the strain gauge at the root of the hair directly, which made an advance in the spatial resolution of the sensor. The sensor could be used in many 


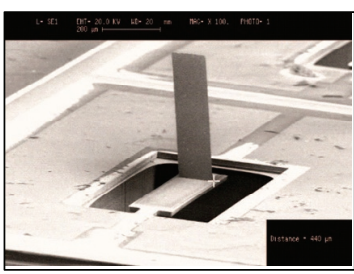

(a)

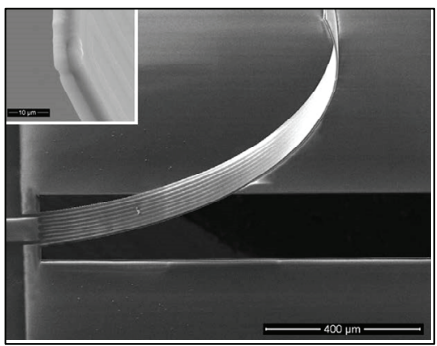

(e)

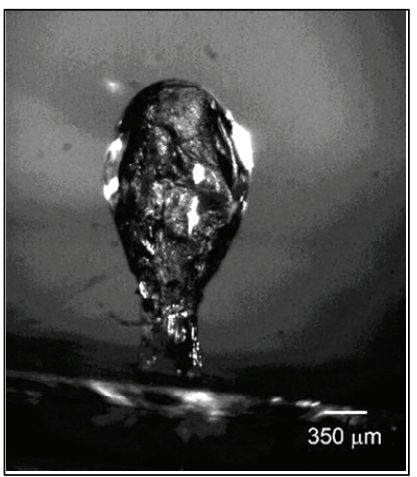

(h)

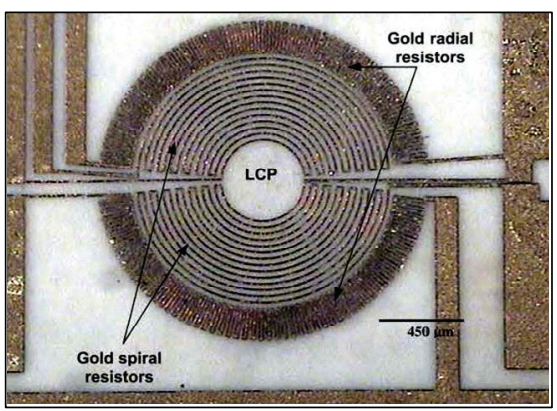

(j)

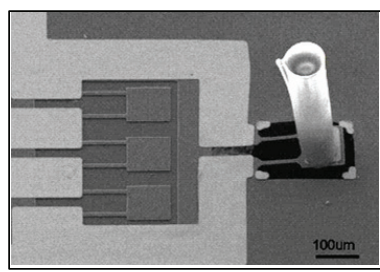

(b)

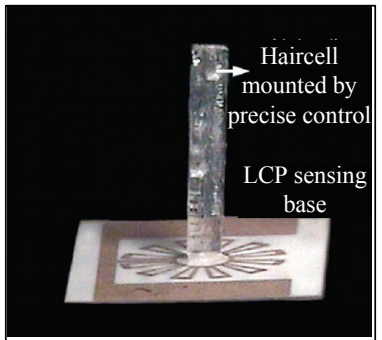

(f)

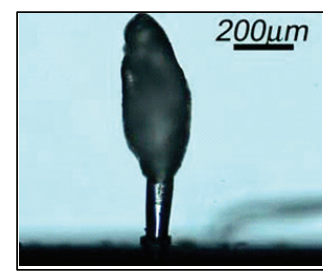

(c)

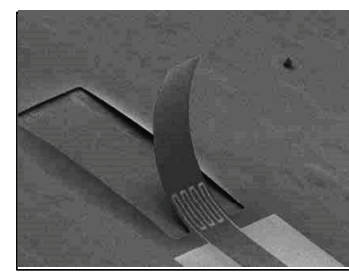

(d)

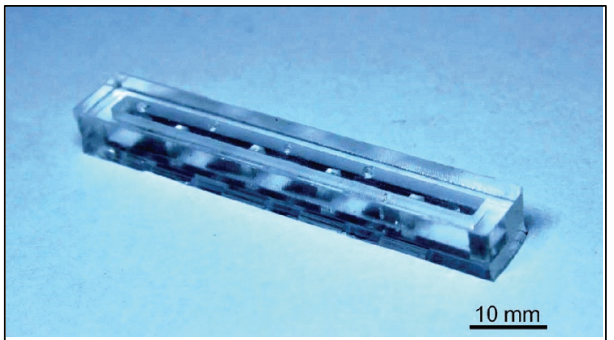

(g)

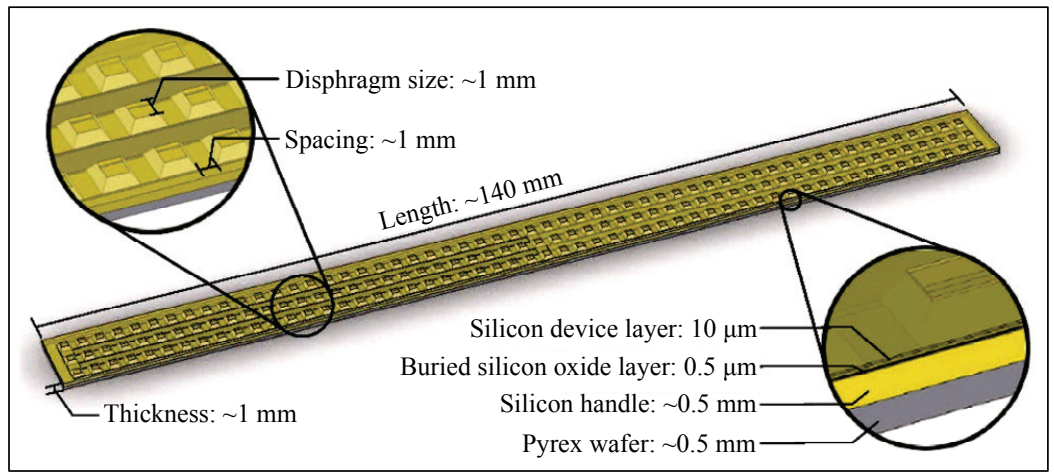

(i)

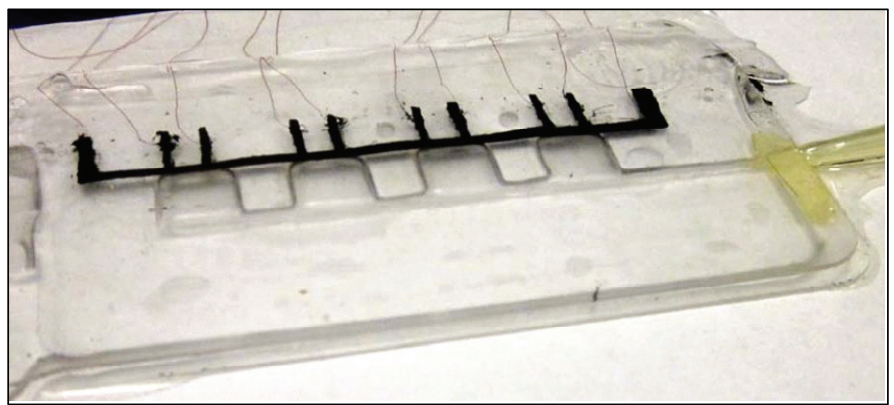

(k)

Fig. 5 Various piezoresistive ALL sensors. (a) Scanning electron micrograph of a single artificial hair cell sensor ${ }^{[18]}$; (b) scanning electron micrograph of an AHC sensor ${ }^{[19]}$; (c) the front-view of a hair sensor after being coated with the hydrogel material ${ }^{[20]}$; (d) scanning electron micrograph on a bent cantilever ${ }^{[21]}$; (e) scanning electron micrograph on a fabricated cantilever ${ }^{[22]}$; (f) an angle view of the complete sensor with hair cell ${ }^{[23]}$; $(\mathrm{g})$ photographic image of the final ALL canal system prototype ${ }^{[24]}$; (h) cupula formed using nanofibrils scaffold has a prolate spheroid shape ${ }^{[25]}$; (i) diagram of the pressure sensor array with basic structure depicted ${ }^{[26]}$; (j) photograph of the pressure sensor array ${ }^{[27]}$; (k) optical microscopic image of a fabricated full-bridge LCP sensor with two radial and two spiral gold piezoresistors ${ }^{[28]}$.

possible flow and temperature conditions and could be assembled on a large scale for a distributed flow sensing $^{[29]}$. In order to further improve the sensitivity and resolution, in 2007, Yang et al. proposed another highly sensitive piezoresistive flow sensor fabricated on a Silicon-On-Insulator (SOI) wafer, the cilium of which was made of photodefinable SU-8 epoxy and adopted a symmetric cylindrical shape. The sensor was used to 
detect the steady-state laminar flow and oscillatory flow with a threshold down to $0.7 \mathrm{~mm} \cdot \mathrm{s}^{-1}$ and showed a high repeatability. The sensor could survive even in harsh environments and show good robustness. However, during the process of photolithography, misalignment is inevitable, which could affect the sensitivity of the sensor $^{[19,30,31]}$. In 2010, they assembled piezoresistive sensors on the surface of a cylindrical polyvinyl chloride (PVC) model and put forward an adaptive beamforming algorithm in order to locate the dipole source in various flow conditions ${ }^{[32]}$.

To match the threshold sensitivity of the integrated fish flow sensory system, McConney et al. created a bio-inspired hydrogel-capped hair sensory system in 2008 using a precision drop-casting method. They added extremely compliant and high-aspect-ratio hydrogel cupula (polyethylene glycol) to the SU-8 hair sensor, as a result of which, the sensitivity of the sensor was enhanced by about two orders of magnitude $\left(2.5 \mu \mathrm{m} \cdot \mathrm{s}^{-1}\right)^{[20]}$.

Unlike the sensors mentioned above whose material was mainly silicon, Qualtieri et al. reported on a kind of ALL sensor in 2011, the key component of which was the stress-driven Aluminium Nitride (AIN) cantilevers. The structures utilizing a multilayered cantilever AlN/Molybdenum (Mo) and a Nichrome 80/20 alloy as piezoresistor were realized by means of micromachining techniques combining optical lithography and etching process. The piezoresistor showed a sensitivity to directionality and low value pressure with a detection threshold of 0.025 bar. The sensor had a promising application prospect because of it stability in almost all flow conditions and simple fabrication ${ }^{[21]}$. Besides, in 2012, they deposited a water resistant parylene conformal coating on the hair cell and developed a biomimetic waterproof $\mathrm{Si} / \mathrm{SiN}$ multilayered cantilever using surface micromachining techniques. The sensor could be used in almost all flow conditions and showed mechanical robustness in high-speed flow and had the capability of discriminating the flow direction at low frequencies ${ }^{[22]}$.

In 2014, Kottapalli et al. developed an artificial SN sensor array composed of a Liquid Crystal Polymer (LCP) membrane, a gold strain gauge and a Si-60 cilium fabricated by stereolithography with a high-aspect ratio of 6.5. The sensor could be used in high temperature and pressure conditions and demonstrated a high sensitivity of $0.9 \mathrm{mV} \cdot\left(\mathrm{m} \cdot \mathrm{s}^{-1}\right)^{-1}$ and $0.022 \mathrm{~V} \cdot\left(\mathrm{m} \cdot \mathrm{s}^{-1}\right)^{-1}$ while detecting air and water flows. The threshold velocity limits were $0.1 \mathrm{~m} \cdot \mathrm{s}^{-1}$ and $15 \mathrm{~mm} \cdot \mathrm{s}^{-1}$, respectively ${ }^{[23]}$. In 2016, they created a canopy-like nanofiber pyramid around the Si-60 polymer cilium and then dropped the casting hydrogel cupula onto the nanofiber scaffold to enhance the sensor for a high sensitivity, low threshold detection limits and a high repeatability. The sensor could sense minute flow velocities within the vortices, which had a wide range of applications in improving the vehicle maneuverability ${ }^{[25]}$.

All sensors mentioned above feature a cilium and a cantilever beam, which bends under the impact of water flow and is sensitive to flow velocity. Except for this, other piezoresistive sensors are mostly planar and the piezoresistors are directly installed on the substrate to detect underwater pressure distribution and variations.

In 2017, Jiang et al. integrated cantilevered flow-sensing elements mainly made of polypropylene and polyvinylidene fluoride (PVDF) layers in a polydimethylsiloxane (PDMS) canal and used it to detect a dipole vibration source. The sensors showed high-pass filtering capability and a pressure gradient detection limit of $11 \mathrm{~Pa} \cdot \mathrm{m}^{-1}$ at the frequency of $115 \mathrm{~Hz}^{[24]}$. Additionally, Fernandez et al. used an array of off-the-shelf pressure sensors to detect cylindrical obstacles of round and square in 2007. The array consisted of hundreds of micro-electromechanical systems (MEMS) pressure sensors which were fabricated on etched Silicon and Pyrex wafers. A strain gauge was mounted on a flexible diaphragm which was a thin $(20 \mu \mathrm{m})$ layer of Silicon attached at the edges of a square Silicon cavity with a width of $2000 \mu \mathrm{m}$ and served as the sensing element with a pressure detection threshold of $1 \mathrm{~Pa}$. The sensors could be used in cloudy and even dark environments ${ }^{[26]}$. In 2012, they presented a 1D array of four sensors with a $15 \mathrm{~mm}$ center-to-center spacing. Each sensor had two key components: a strain-concentrating PDMS diaphragm and a resistive strain gauge made of a conductive carbon-black PDMS composite. The resolution of it was 1.5 Pa. Because of the irreversible breaking of parts of the carbon chains, external pressure stimulus with larger amplitude or lower frequency caused an increased upward drift in the resistance of the strain gauge. Due to 
viscoelastic creep, the repeatability was about $22 \%$ of the peak amplitude. The sensor demonstrated flexibility, chemical robustness, and waterproofing because of the properties of carbon-black-PDMS composite which had a great potential to be applied to ALL as a transduction element ${ }^{[27]}$.

To perform underwater surveillance, Kottapalli et al. developed an array of polymer MEMS pressure sensors fabricated with a $\mathrm{Cr}(20 \mathrm{~nm}) / \mathrm{Au}(700 \mathrm{~nm})$ thick gold layer sputtered on a flexible substrate and LCP serving as the sensing membrane material. Installed on curved surfaces of the underwater vehicle bodies, the sensors detected underwater objects by sensing the pressure variations. Compared with Silicon-based hair vertical structures or thin metal cantilever beams, it showed a better sensitivity of $14.3 \mu \mathrm{V} \cdot \mathrm{Pa}^{-1}$ and a better resolution of $25 \mathrm{~mm} \cdot \mathrm{s}^{-1}$ in water flow sensing. The sensors also proved the superior performance of LCP as sensing materials for harsh deep sea environments because of its excellent chemical robustness, hermeticity and fracture strength ${ }^{[28]}$.

\subsubsection{Piezoelectric ALL sensors}

Piezoelectricity refers to the electric charge generated on the surface of some certain materials while subjected to external forces. This effect inspires another kind of ALL sensors which is able to sense environment by collecting the electric information. Fig. 6 shows various piezoelectric ALL sensors mentioned below.

For the purpose of environmental perception and obstacle avoidance, Asadnia et al. used floating bottom electrodes to design an array of $\mathrm{Pb}\left(\mathrm{Zr}_{0.52} \mathrm{Ti}_{0.48}\right) \mathrm{O}_{3}$ thin-film piezoelectric pressure sensors in 2013. Packaged into an array of 25 sensors on a flexible liquid crystal polymer substrate patterned with gold interconnects, the array was used to locate a vibrating sphere dipole in water and showed a resolution of $3 \mathrm{~mm} \cdot \mathrm{s}^{-1}$ in detecting oscillatory flow velocity. Besides, the sensors had many advantages such as self-powered, miniaturized, light-weight, low-cost, robust and applied in harsh environments ${ }^{[33]}$. In 2015, they optimized the sensor by mounting a stereolithographically fabricated polymer hair cell on microdiaphragm with floating bottom electrodes. The sensor demonstrated a high-pass filtering nature with a cut-off frequency of $10 \mathrm{~Hz}$, a high sensi- tivity of $22 \mathrm{mV} \cdot\left(\mathrm{mm} \cdot \mathrm{s}^{-1}\right)^{-1}$ and a resolution of $8.2 \mathrm{~mm} \cdot \mathrm{s}^{-1}$ in water flow detection ${ }^{[36]}$. In 2016 , they reported the development of a new class of miniature all-polymer flow sensors with an artificial ciliary bundle fabricated by combining bundled PDMS micro-pillars with graded heights and electrospinning PVDF piezoelectric nanofiber tip links. By means of precision drop-casting and swelling processes, a dome-shaped hyaluronic acid hydrogel cupula encapsulating the artificial hair cell bundle was formed. The sensors achieved a sensitivity of $300 \mathrm{mV} \cdot\left(\mathrm{mm} \cdot \mathrm{s}^{-1}\right)^{-1}$ and a threshold detection limit of $8 \mu \mathrm{m} \cdot \mathrm{s}^{-1}$ respectively. The sensor could perform passive sensing that enables underwater robots and vehicles to achieve hydrodynamic artificial vision, super mobility and extreme control. Miniaturized size, biocompatibility, and ability to operate without an external power source discriminated them from other sensors and extended their applications to biomedical devices and microfluidics ${ }^{[34]}$.

In 2011, Abdulsadda et al. proposed a novel ALL sensors utilizing the inherent sensing capability of Ionic Polymer-Metal Composites (IPMCs). An IPMC consisted of three layers, with an ion-exchange polymer membrane sandwiched by metal electrodes. Detectable electrical signals were produced under the impact of external forces. The IPMC flow sensors were used to localize dipole sources $4-5$ body away and demonstrated a threshold detection limit of less than $1 \mathrm{~mm} \cdot \mathrm{s}^{-1}$. However, the capability of signal acquisition of the sensor needed to be improved ${ }^{[35]}$.

\subsubsection{Capacitive ALL sensors}

Owing to the high sensitivity and low power consumption, capacitance principle has been widely used in many different types of sensors. The key component is the capacitive readout which has the capability of converting external stimulus into capacitance changes, which provides an effective way to detect underwater pressure and flow velocity. Like the piezoresistive sensors, the hair attached to the membrane will respond to the impact from the local flow. And then the membrane reflects and changes the distance or gap between the electrodes. As a result, the change in capacitance is quantitatively related to the external impact. Fig. 7 shows various capacitive ALL sensors mentioned 


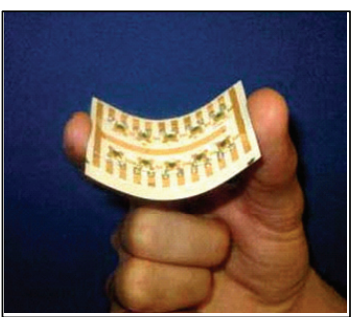

(a)

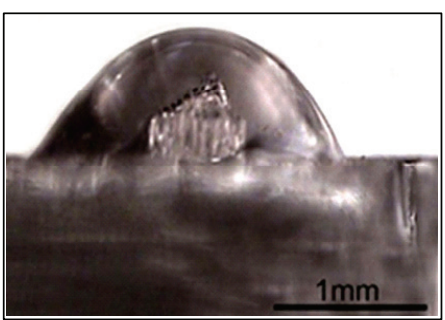

(b)

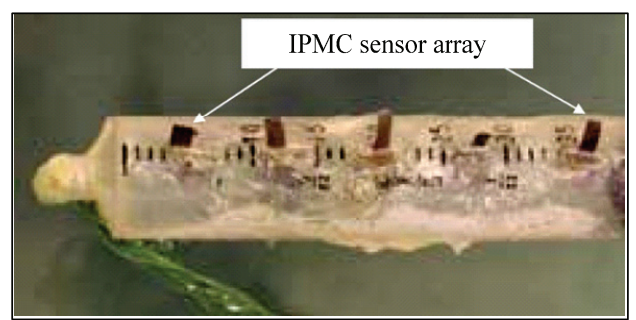

(c)

Fig. 6 Various piezoelectric ALL sensors. (a) Array of 2 by 5 piezoelectric sensors on flexible LCP substrate ${ }^{[33]}$; (b) icroscopic side-view image of the sensor showing the hydrogel cupula and the PDMS pillars with height gradient ${ }^{[34]}$; (c) the IPMC-based lateral line prototype $^{[35]}$.

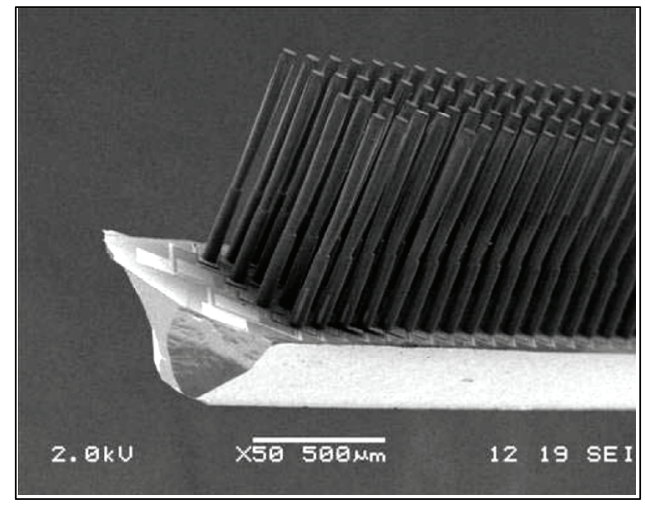

(a)

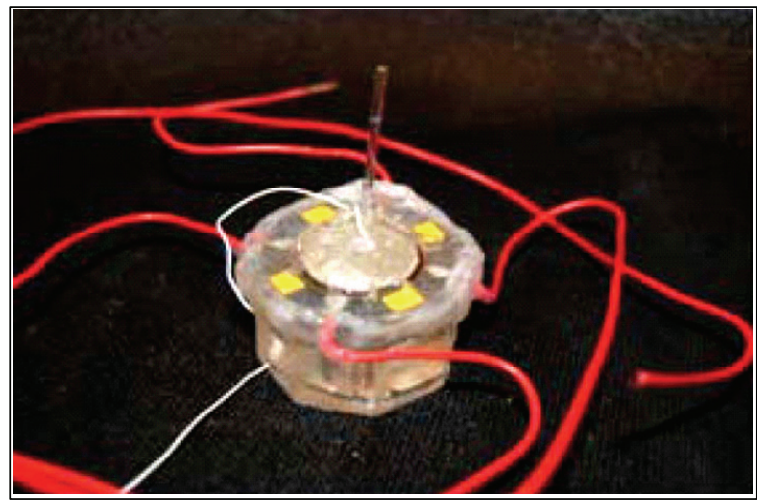

(b)

Fig. 7 Various capacitive ALL sensors. (a) Scanning Electron Microscope image of actual sensors ${ }^{[37]}$; (b) top views of the ALL sensor ${ }^{[38]}$.

below.

In 2007, Krijnen et al. reported developments in hair sensors based on mechanoreceptive sensory hairs of crickets using artificial polysilicon technique to form Silicon Nitride suspended membranes and SU-8 polymer processing to form hairs with the diameter of about $50 \mu \mathrm{m}$ and the length of $1 \mathrm{~mm}$. The membranes had thin chromium electrodes on top which formed variable capacitors and the sensitivity of the sensor is $1.39 \mathrm{pF} \cdot \mathrm{rad}^{-1}$. However, the capacitor structure needed further optimization for a better resolution ${ }^{[37,39]}$. In 2010, they realized the dense arrays of fully supported flexible SU-8 membranes with integrated electrodes underneath which supported cylindrical hair-like structures on the top. They insulated the electrodes from liquid to prevent short circuit or electrolysis. The sensor demonstrated good flexibility. While used in air flow detection, the mechanical sensitivity at the frequency of $115 \mathrm{~Hz}$ was $0.004 \mathrm{rad} \cdot(\mathrm{m} \cdot \mathrm{s})^{-1[40]}$.

Another capacitive whisker sensor inspired by seal vibrissae was developed by Stocking et al. to measure the flow velocity and detect the direction in 2010 . They mounted a rigid artificial whisker on a novel cone-incone parallel-plate capacitor base which was covered by a PDMS membrane. Numerical simulation predicted the change of capacitor output signal in a range of $1 \mathrm{pF}$ when the flow velocity varied from $0 \mathrm{~m} \cdot \mathrm{s}^{-1}$ to $1.0 \mathrm{~m} \cdot \mathrm{s}^{-1}$ in various flow conditions. However, the predicted baseline capacitance and signal response were different from experimental results for some reasons, which needed to be addressed by optimizing the structure ${ }^{[38]}$.

\subsubsection{Optical ALL sensors}

Optical principles have also been used to develop ALL sensors. Fig. 8 shows various optical ALL sensors mentioned below. Klein et al. made a great breakthrough in this area in 2011. The artificial canal neuromasts segment they developed consisted of a transparent silicone bar which had the same density of water and an infrared light emitting diode at one end of the silicone bar. To detect the fluid motion, light, leaving the opposite end of the silicone bar, illuminated an optical fiber 


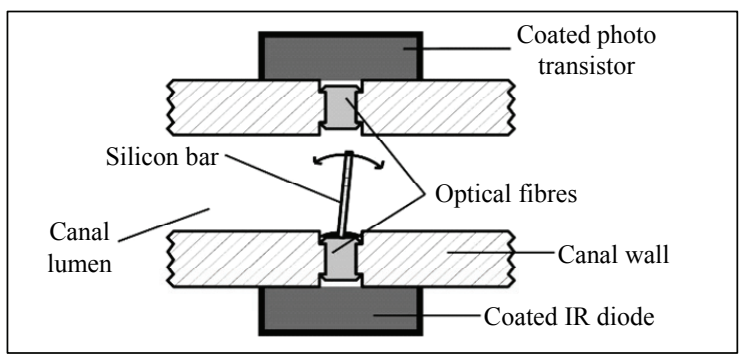

(a)

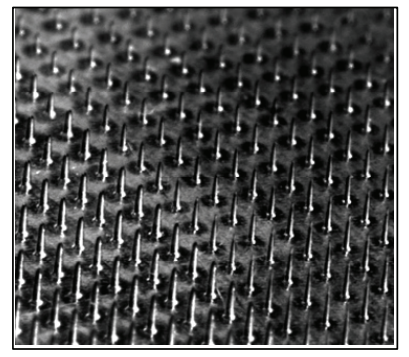

(b)

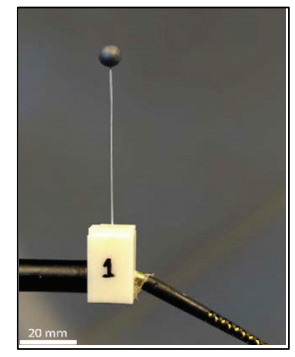

(c)

Fig. 8 Various optical ALL sensors. (a) Scheme of an artificial $\mathrm{CN}^{[41]}$; (b) scanning-electron microscope image of a pillar array ${ }^{[42]}$; (c) the photograph of the all-optical sensor ${ }^{[43]}$.

that was connected to a Surface Mounted Devices (SMD) phototransistor and the output was amplified and converted to be stored on a computer. The sensors could be used to detect water and air motions caused by a stationary vibrating sphere or a passing object and vortices caused by an upstream cylinder. However, due to different Reynolds numbers, lager velocity was needed in air for signal acquisition. According to the acquired information, they calculated the bulk flow velocity and the size of the cylinder producing the vortices. The detection limit in water flow was $100 \mu \mathrm{m} \cdot \mathrm{s}^{-1}$. The sensors were not exposed to environments so they cause minimal effects on flow field and were less damaged physically. Due to their structural characteristics, the sensitivity, frequency response and dynamic amplitude range can be changed easily ${ }^{[41]}$.

Another method was put forward by Große and Schröder in 2009. The key component of the sensor was flexible micro-pillars which protruded into local flows and bent when subjected to exerted drag forces. The pillar was fabricated from the elastomer PDMS and the deflection was measured by means of optical methods. They have also carried out experiments to demonstrate the low drift of the sensor within the range of measurement and the repeatability was within $\pm 1.5 \%$ F.S. The structure could be extended to detecting flows at high Reynolds numbers ${ }^{[42]}$.

In 2018, Wolf et al. presented an all-optical 2D flow velocity sensor consisting of optical fibres inscribed with Bragg gratings supporting a fluid force recipient sphere. The sensor demonstrated a threshold of $5 \mu \mathrm{m} \cdot \mathrm{s}^{-1}$ at a low frequency and $5 \mathrm{~mm} \cdot \mathrm{s}^{-1}$ at resonance with a typical linear dynamic range of $38 \mathrm{~dB}$ at $100 \mathrm{~Hz}$ sampling. Additionally, the artificial neuromast is capable of detecting flow direction within a few degrees $^{[43]}$

\subsubsection{Hot-wire ALL sensors}

The most important part of Hot-Wire Anemometer (HWA) is a heated wire placed in the air. While air or water flows through it, heat loss leads to changes in temperature and resistance. Therefore, we can measure the velocity by detecting electrical signals. Fig. 9 shows various hot-wire ALL sensors mentioned below.

In 2006, Yang et al. developed a surface-micromachined, out-of-plane ALL sensor array using the principle of thermal HWA. Inspired by the SNs of fish, the hot wire was lifted from the substrate by two pointed heads. They utilized photolithography technique to fabricate the sensor in plane and assembled it outside by three-dimensional magnetic assembly. The sensor afterwards was used to track the position of a vibrating dipole source and exhibited a threshold of $0.2 \mathrm{~mm} \cdot \mathrm{s}^{-1}$ with a bandwidth of $1 \mathrm{kHz}$ and the drift of zero outputs was negligible $e^{[44,46,47]}$.

Liu et al. proposed a novel micromachined hot-film flow sensor system realized by using a film depositing process and a standard printed circuit in 2009. They preprinted the sensor electrodes and electronic circuits on a flexible substrate of polyimide and utilized $\mathrm{Cr} / \mathrm{Ni} / \mathrm{Pt}$ as the sensing element with a resistance temperature coefficient around $2000 \mathrm{ppm} \cdot \mathrm{K}^{-1}$. The resolution of this sensor was $0.1 \mathrm{~m} \cdot \mathrm{s}^{-1}$ and the repeatability was $\pm 0.3 \%$ F.S. The sensor system showed good mechanical characteristics and sensing capability and had the advantages of simple structure, low-cost, flexibility and easily 


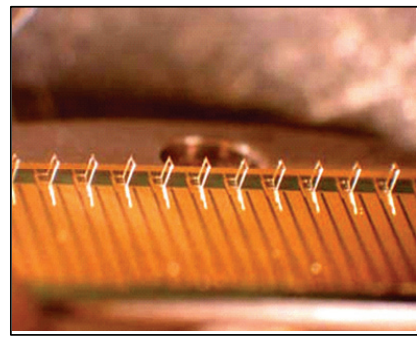

(a)

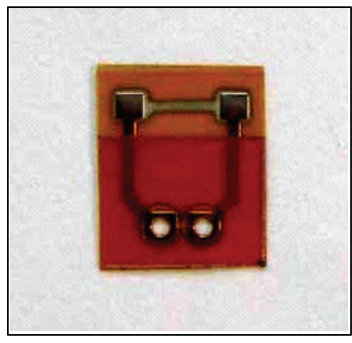

(b)
Fig. 9 Various hot-wire ALL sensors. (a)An optical micrograph of an $\mathrm{ALL}^{[44]}$; (b) a sensor unit ${ }^{[45]}$.

attached on object surface ${ }^{[45]}$.

\subsection{ALL sensors placement optimization}

With the development of ALL sensor units, more efforts have been devoted to sensors placement optimization in order to simulate the fish lateral line to a greater extent. Verma et al. used a larva-shaped swimmer exposed in disturbances induced by oscillating, rotating and cylinders to conduct experiments in 2020. Combining Navier-Stokes equations with Bayesian experimental design and with a purpose of detecting the location of the source, they presented that shear sensors should be installed on the head and the tail while pressure sensors should be distributed uniformly along the body and intensively on the head, which is similar to real fish lateral line ${ }^{[48]}$. In 2019, Xu et al. put forward an optimal weight analysis algorithm combined with feature distance and variance evaluation and 3 indexes to evaluate the performance of the sensor array. They also briefly discussed the optimal number of sensors ${ }^{[49]}$. This work has provided new ideas for studies in ALL sensors distribution optimization in the future.

In this section, we have presented ALL sensors based on different sensing mechanisms and briefly introduced works in sensors placement optimization. Table 1 summarizes the parameters of the ALL sensors, including transduction mechanism, processing technique, material and sensitivity. Though great progress has been made in the design and fabrication of ALL sensors, the sensors mentioned above are mostly simple imitations of the real fish lateral line and have a long way to go in sensitivity and repeatability. Firstly, the sensitivity of a single sensory unit can be further improved. Additionally, the neuromasts distribution of fish is continuously optimized in evolution. Thus, it is necessary for us to find out the optimal distribution of ALL sensors according to different shapes of robotic fish in order to simulate the lateral line to the maximum extent and develop a complete ALL system based on existing local sensor arrays. Last but not least, we need more efficient signal processing and agent decision algorithm methods to take full advantage of the information acquired by the ALL system and make decisions like a living body. ALL systems suchlike have great potentials for future oceanographic research.

\section{Hydrodynamic environment sensing and vortices detection}

Due to the limitations of existing measurements methods in complex natural flows and the emergence of the above mentioned various types of sensors, scientists have started to use ALL systems consisting of these sensors to obtain information from fluid environment. Efforts are devoted into the following fields: flow field characteristics identification, flow velocity and direction detection, vortex street properties detection. Carriers boarded with ALL mentioned in this section are shown in Fig. 10.

\subsection{Flow field characteristics identification}

In 2013, Salumäe and Kruusmaa presented that the robotic fish boarded with 5 pressure sensors (Fig. 10b) was able to identify the flow regimens (uniform flow and periodic turbulence) according to the pressure around the underwater vehicle. Based on the results, they showed that artificial lateral line can be used for controlling the motion of robots with respect to the flow. This navigation method could be used on any other underwater vehicles, which is especially valuable for the stability of vehicles in turbulent flows ${ }^{[51]}$.

In 2018, they used the ALL probe consisting of 11 piezoresistive pressure sensors (Fig. 10d) to capture hydrodynamic information. The probe detected an average flow velocity in turbulent flows which was comparable to the results measured practically. According to the characteristics of the fluid, the flows were classified by comparing the probability distribution of turbulent pressure fluctuation. The classification of flow fields is very important to the control and navigation of underwater vehicles ${ }^{[53]}$. 
Table 1 Summary of ALL sensors

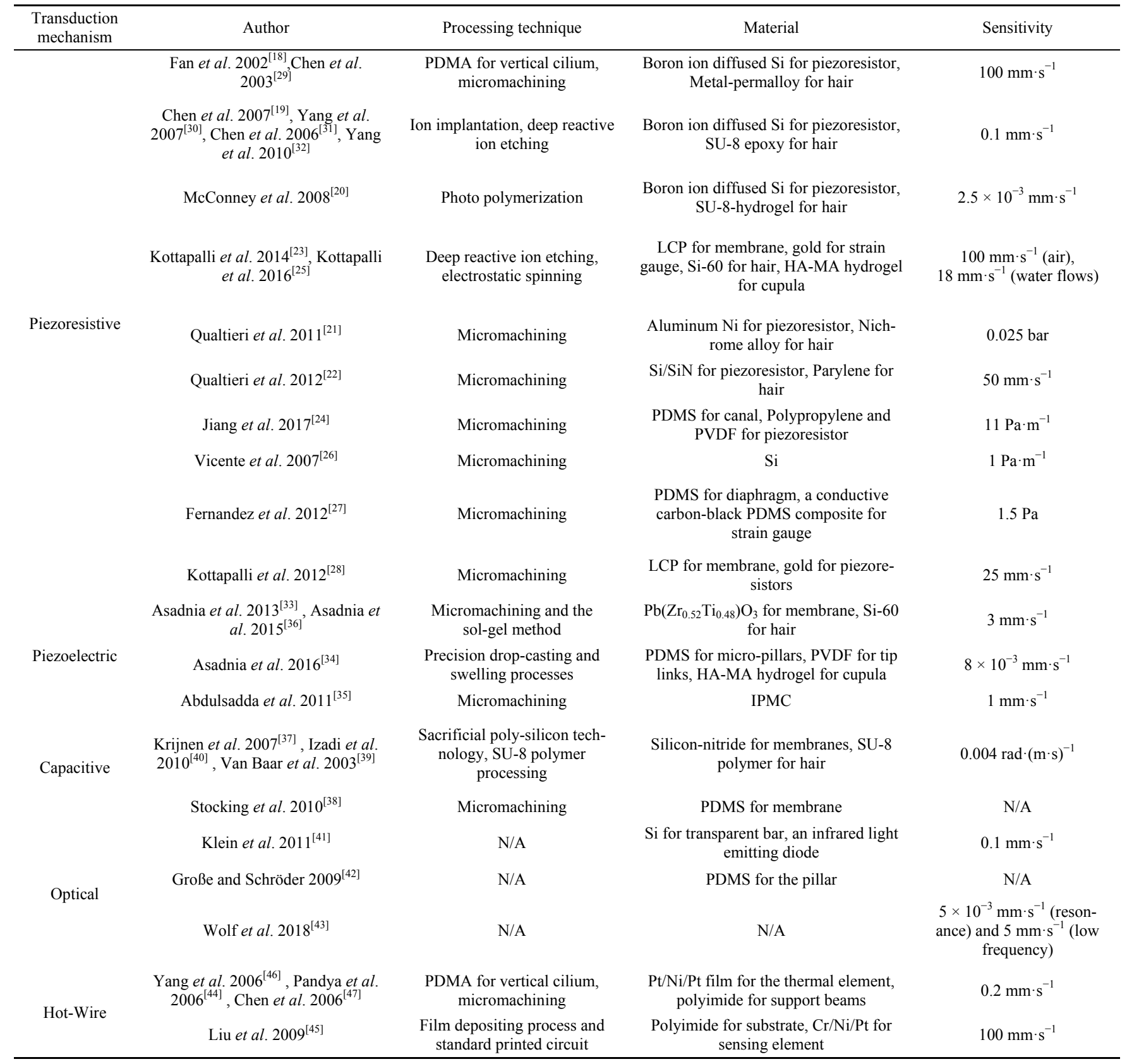

Liu et al. also made great contribution in flow field characteristics identification. In 2019, they proposed an improved pressure distribution model to simulate the pressure around the ALL consisting of 23 pressure sensors (Fig. 10e) and then established a visualized pressure difference matrix to identify flow field in different conditions. A four-layer convolutional neural network model was constructed to evaluate the accuracy of this method $^{[54]}$.

\subsection{Flow velocity and direction detection}

In 2013, Salumäe and Kruusmaa proved that the flow speed can be estimated only by the average pressure on the sides of the robotic fish (Fig. 10b). They put forward a fitted formula representing the relationship between the average pressure variance and the flow speed based on Bernoulli formula. Besides, they also reported that the flow direction could be detected because the pressure on the side which the probe turned 


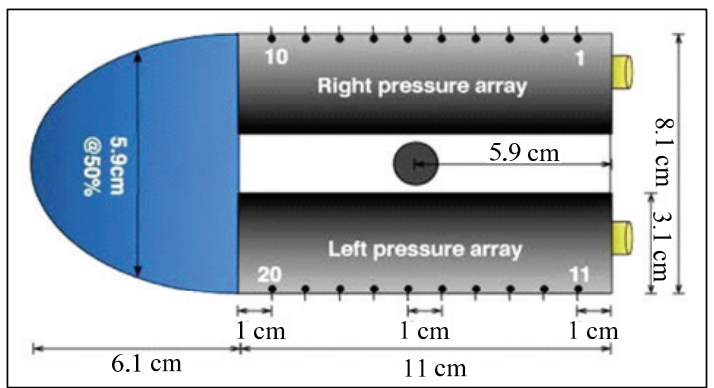

(a)

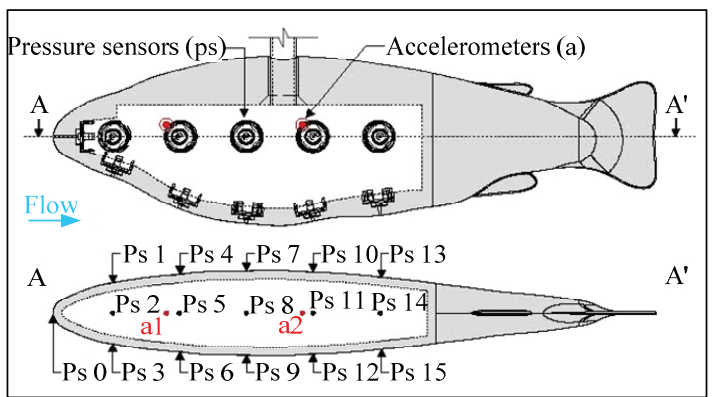

(c)

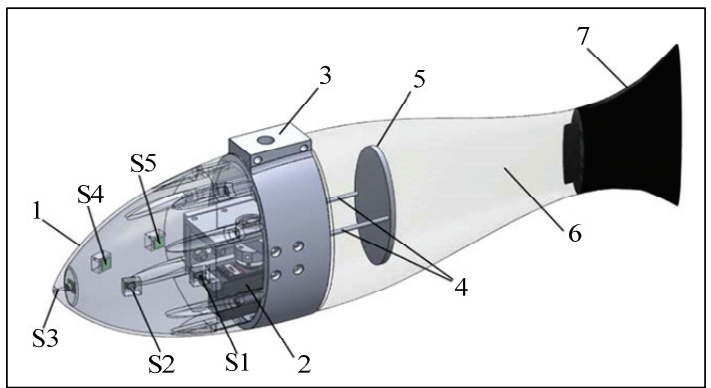

(b)

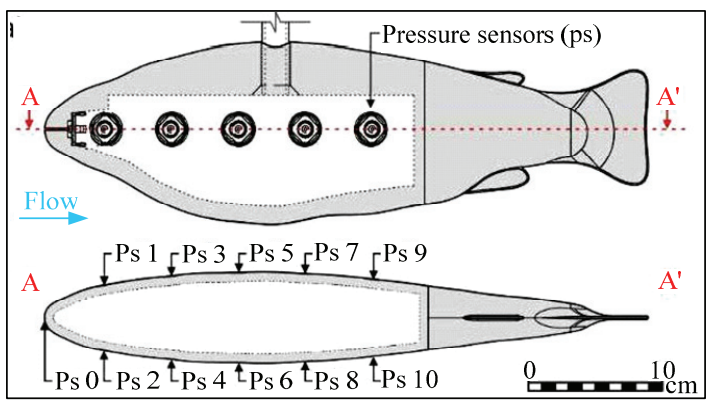

(d)

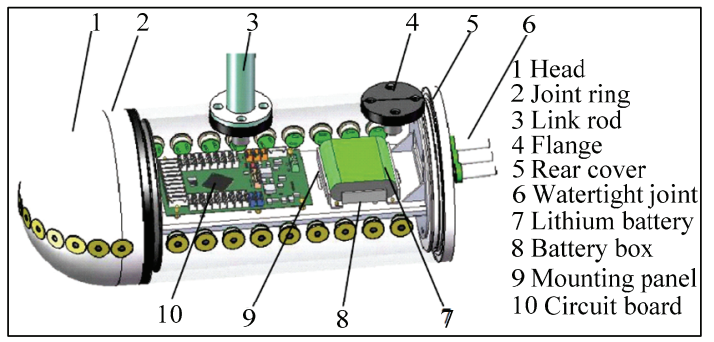

(e)

Fig. 10 Different carriers boarded with ALL mentioned in section 4. (a) A schematic diagram of the sensor platform used in Ref. [50]. (b) CAD view of the robot used in Ref. [51]: 1, rigid head of the robot; 2, servo-motor; 3, middle part for holding the head and the tail; 4, steel cables; 5, actuation plate; 6, compliant tail; 7, rigid fin; S1-S5, pressure sensors. (c) Location of the 16 pressure sensors and 2 accelerometers in the ALL Probe used in Ref. [52]. (d) Illustration of the lateral line probe used for field measurements showing shape and sensor distribution in Ref. [53]. (e) A 3D model of the carrier in Ref. [54].

towards the flow was higher. The detection of flow velocity and flow direction can help the robotic fish to identify the flow field. Acquisition of flow information is quite important for robotic fish for their navigation underwater $^{[51]}$.

In the same group, they presented a new way for flow speed estimation with the help of ALL probe consisting of 10 piezoresistive pressure sensors (Fig. 10c) without sensor calibration in 2015, which is of great convenience. Induced by the interactions between fluid and the robot body, fluctuations in the pressure field around the body could be detected by the ALL probe.
Based on Bernoulli formula likewise, they introduced a semiempirical resampling process. Compared with results measured by an acoustic Doppler velocimeter in a vertical slot fishway, the accuracy of this method was validated $^{[52]}$.

Besides, Strokina et al. made great progress in natural flow measurements using an ALL probe combined with signal processing methods in 2016. The probe is the same as showed in Fig. 10c. They proved that information acquired by the probe was transformed into two important hydrodynamic primitives, bulk flow velocity and bulk flow angle via canonical signal 
transformation and kernel ridge regression. Moreover, they showed that this method was effective not only when the sensor was parallel to the flow, but also in the condition that the angular deviation was large. While used in a natural river environment, the method had an error of $14 \mathrm{~cm} \cdot \mathrm{s}^{-1[55]}$. In 2016, they presented a new method to estimate the flow velocity ranging from $0 \mathrm{~m} \cdot \mathrm{s}^{-1}$ to $1.5 \mathrm{~m} \cdot \mathrm{s}^{-1}$. They collected time-averaged flow velocity and pressure acquired by the ALL in highly turbulent flow and put forward a signal processing approach combining Pearson product-moment correlation coefficient features and artificial neural network ${ }^{[56]}$. This method is potential to interpret the underwater preferences of fish in real environment.

Additionally, Liu et al. in 2020, based on a fitting method and a back propagation neural network model, successfully predicted the flow velocity and direction and the moving velocity ${ }^{[57]}$. The employment of methods of machine learning provided another possibility to sense hydrodynamic information for further study.

\subsection{Vortex street properties detection}

Vortex street is one of the most common flows in nature, such as wakes of a swinging tail and flows behind obstacles. The detection of vortex street characteristics and the estimation of upstream objects are valuable for robot fish to navigate in the natural flow environment. In 2006, Yang et al. used an ALL system with 16 HWA sensors to study the spatial velocity distribution of Kármán vortex street and visualized the velocity distribution of Kármán vortex street generated by a cylinder for the first time ${ }^{[46]}$.

Ren and Mohseni theoretically studied the perception of vortex streets using real lateral line in 2010. Based on potential flow theory, they constructed the model of flow field around the fish, and then explained how the fish captured the characteristics of vortices with the help of lateral line CNs. The model were applied to estimate the range of the vortex, transmission speed, direction, distance between the vortex streets and distance between the fish and the vortex street ${ }^{[58]}$.

In 2011, Klein and Bleckmann used an artificial canal equipped with optical flow sensors which have been presented in section 3 (Fig. 8a). They have demonstrated the capability of the ALL canals to detect the vibrating sphere. Additionally, vortices generated by an upstream cylinder were also detected. Based on the hydrodynamic information acquired by the ALL canal, they succeeded in calculating the flow velocity and the size of the cylinder ${ }^{[41]}$.

In 2012, Venturelli et al. used digital particle image velocimeter to visualize the flow state and a rigid body equipped with 20 pressure sensors (Fig. 10a) parallel distributed to acquire flow field information and then applied time and frequency domain methods to describe hydrodynamic characteristics in steady and unsteady flows respectively. The array of pressure sensors showed a capability of discriminating vortex streets from steady flows and detecting the position and direction of the body relative to the incoming flow. A series of hydrodynamic parameters were also calculated, such as vortex shedding frequency, vortex travelling speed and downstream distance between vortices ${ }^{[50]}$.

Free et al. presented a method to estimate the parameters of vortices in 2017. They used a straight array of 4 pressure sensors to sense a spiral vortex and a square array to sense a Kármán vortex street. Based on potential flow theory and Bernoulli principle, the measurement equation was incorporated in a recursive Bayesian filter, as a consequence of which, the position and strength of vortices have been successfully estimated. Moreover, they identified an optimal path for underwater vehicles to swim through a Kármán vortex street using empirical observability. Experiments demonstrated the effectiveness of the closed-loop control ${ }^{[59]}$. Based on the results above, in 2018, they installed the array on a Joukowski foil and detected Kármán vortex streets nearby. With the help of trajectory-tracking feedback control, the robotic foil performed fish-like slaloming behavior in a Kármán vortex street ${ }^{[60]}$.

In this section, we have focused on the application of ALL systems in detecting flow characteristics. Table 2 as follows lists different projects mentioned above and related ongoing studies. Existing results are mainly based on static ALL sensors and experiments are conducted in laboratory environment. The characteristics of flow which can be detected are also limited. For further study, with the improvement of ALL systems, we can pay more attention to natural environment experiments, where the complexity of the water environment and the 
complex movements of the robot fish make it more difficult for perception.

\section{ALL based dipole source detection}

The localization ability of underwater objects with the help of ALL systems can effectively improve the viability of robotic fish in underwater environment. In addition to the anti-Kármán vortex street, a near-dipole flow field is generated by the fin while fish swims. This can also explain how predators capture preys ${ }^{[61]}$. Dipole oscillation source detection has become a common problem in hydrodynamics and the development of ALL. While the dipoles are vibrating or moving in a certain way, the pressure and the flow speed will change accordingly. By measuring the information with the help of ALL, we can infer the motion of the object for further study. Carriers boarded with ALL mentioned in this section are shown in Fig. 11.

Tang et al. represented an array of 8 pressure sensors installed on the surface of an underwater vehicle (Fig. 11a) inspired by lateral line for near-field detection in 2019. The pressure field generated by vibrating sphere which was simulated as an underwater pressure source was derived by means of linearizing the kinematic and dynamic conditions of the free surface wave equation. The pressure field detected by the ALL was consistent with simulation results ${ }^{[62]}$.

In 2007, Yang et al. used an array of AHCs (Fig. 11b) whose sensory unit has been introduced in section 3 (Fig. 5b) to locate and track the dipole source. As for mapping the pressure field produced by the dipole source, the array performed well and the results was consistent in experiments and theory. As for tracking the trail, a cylinder was put in a steady flow with the speed of $0.2 \mathrm{~m} \cdot \mathrm{s}^{-1}$ to simulate the fluid trail which was dominated by Kármán vortex street ${ }^{[30]}$.

In 2010, the same group developed an ALL by wrapping 15 pressure sensors around a cylinder (Fig. 11c) to mimic real fish and proved its localization capability. They used a beamforming algorithm to image hydrodynamic events in a 3D domain. Consequently, the ALL sensors was demonstrated to be able to localize a dipole source and a tail-flicking crayfish accurately in varieties of conditions $^{[32]}$.

$$
\text { Additionally, Asadnia et al. packaged }
$$

$\mathrm{Pb}\left(\mathrm{Zr}_{0.52} \mathrm{Ti}_{0.48}\right) \mathrm{O}_{3}$ thin-film piezoelectric pressure sensors for underwater sensing in 2013. The array of 2 by 5 sensors has been showed in section 3 (Fig. 6a). While the dipole was driven at the frequency of $15 \mathrm{~Hz}$ and moved parallel to the array, by measuring the maximum peak-to-peak output of the sensors, they approximately estimated the position of the dipole source. To detect the flow velocity generated by the dipole source, the array showed a resolution of $3 \mathrm{~mm} \cdot \mathrm{s}^{-1[33]}$.

Abdulsadda et al. put forward an array of ALL piezoelectric sensors based on the sensing capability of IPMC in 2011. The signals were processed through a widely-used neural network, which was similar to the biological mechanism. The ALL has been presented in the section 3 as well (Fig. 6c). Experiments proved that the ALL could effectively locate the dipole source and the flapping tail. Moreover, the more sensors was used, the more precise the results were ${ }^{[35]}$. In 2013, based on an analytical model of flow field produced by the dipole source, they presented another two schemes, Gauss Newton (GN) algorithms which were used to solve the nonlinear estimation problem by means of linear iteration and Newton Raphson (NR) algorithms which were used to solve the nonlinear equation under the condition of first-order optimality, to locate the dipole source and estimate amplitude and direction of the vibration. Additionally, they improved the design of intra-sensor spacing (Fig. 11f) of the ALL by analysis based on Cramer-Rao Bound (CRB). With 19 dipole sources placed along an ellipsoidal track, the simulation and experiment results both proved the accuracy of this model ${ }^{[65,69,70]}$. In order to reduce the influence of uncertainty in measurements and flow model and thus identify a vibrating dipole accurately, Ahrari et al. developed a specialized bi-level optimization methodology to optimize the design parameters of the ALL ${ }^{[66]}$ (Fig. 11g).

In the aspect of using hot-wire flow sensors to detect the dipole source, Pandya et al. made great contributions in 2006. Section 3 has introduced the hot-wire ALL developed by them (Fig. 9a). To make full use of the ALL, they reported on the implementation of a algorithm consisting of the template training approach and the modeling approach based on Minimum Mean-Squared Error (MMSE) algorithm in order to locate and track a vibrational dipole source ${ }^{[44]}$. 
Table 2 Classification of existing studies in hydrodynamic environment sensing and vortices detection

\begin{tabular}{|c|c|c|c|}
\hline Project & Author & ALL sensors & $\begin{array}{c}\text { Laboratory experiment/Natural } \\
\text { environment experiment }\end{array}$ \\
\hline \multirow{4}{*}{$\begin{array}{l}\text { Flow field characte- } \\
\text { ristics identification }\end{array}$} & $\begin{array}{l}\text { Salumäe and Kruusmaa } \\
\qquad 2013^{[51]}\end{array}$ & 5 pressure sensors (Intersema MS5407-AM) & Laboratory experiment \\
\hline & Tuhtan et al. $2018^{[53]}$ & 11 pressure sensors (SM5420C-030-A-P-S) & Laboratory experiment \\
\hline & Liu et al. $2019^{[54]}$ & 23 pressure sensors (MS5803-07BA) & Laboratory experiment \\
\hline & $\begin{array}{l}\text { Salumäe and Kruusmaa } \\
\qquad 2013^{[51]}\end{array}$ & 5 pressure sensors (Intersema MS5407-AM) & Laboratory experiment \\
\hline \multirow{2}{*}{$\begin{array}{l}\text { Flow velocity and } \\
\text { direction detection }\end{array}$} & $\begin{array}{l}\text { Fuentes-Pérez et al. } 2015^{[52]} \\
\quad \text { Tuhtan et al. } 2016^{[56]}\end{array}$ & $\begin{array}{l}16 \text { pressure sensors (SM5420C-030-A-P-S) and } 2 \text { three-axis } \\
\text { accelerometers (ADXL325BCPZ) }\end{array}$ & Laboratory experiment \\
\hline & Strokina et al. $2016^{[55]}$ & $\begin{array}{l}16 \text { pressure sensors (SM5420C-030-A-P-S) and } 2 \text { three-axis } \\
\text { accelerometers (ADXL325BCPZ) }\end{array}$ & $\begin{array}{l}\text { Laboratory experiment and natural } \\
\text { environment experiment }\end{array}$ \\
\hline \multirow{7}{*}{$\begin{array}{l}\text { Vortex street proper- } \\
\text { ties detection }\end{array}$} & Liu et al. $2020^{[57]}$ & 23 pressure sensors (MS5803-07BA) & Laboratory experiment \\
\hline & Yang et al. $2006^{[46]}$ & 16 HWA sensors & Laboratory experiment \\
\hline & Ren et al. $2010^{[58]}$ & \multicolumn{2}{|l|}{ Theoretical model } \\
\hline & Klein et al. $2011^{[41]}$ & Optical sensors & Laboratory experiment \\
\hline & Venturelli et al. $2012^{[50]}$ & 20 pressure sensors & Laboratory experiment \\
\hline & $\begin{array}{l}\text { Salumäe and Kruusmaa } \\
\qquad 2013^{[51]}\end{array}$ & $\begin{array}{l}5 \text { pressure sensors (Intersema } \\
\text { MS5407-AM) }\end{array}$ & Laboratory experiment \\
\hline & $\begin{array}{l}\text { Free } \text { et al. } 2017^{[59]}, \text { Free } \text { et al. } \\
\qquad 2018^{[60]}\end{array}$ & An array of 4 pressure sensors & Laboratory experiment \\
\hline
\end{tabular}

In 2010, by detecting the parallel and the perpendicular velocity components, Dagamseh et al. used an array of hair flow sensors to reconstruct the velocity field induced by the dipole source in the air and measure the distance ${ }^{[71-74]}$. In 2013, they employed beamforming techniques and improved the performance of the sensor $\operatorname{array}^{[75]}$.

Inspired by the sensory abilities of lateral line, Zheng et al. developed an ALL composed of 9 underwater pressure sensors forming a cross (Fig. 11d) to locate a dipole source in 2018. For the sake of handling nonlinear pattern identification problem, they adopted the method of generalized regression neural network which performed well on condition that the array is below $13 \mathrm{~cm}$ away from the dipole source ${ }^{[63]}$. Besides, the same group develops another ALL composed of 9 pressure sensors in a straight line (Fig. 11e) to locate the dipole source. Lin et al. modelled the pressure field of the dipole source and obtained the position through least square method by means of the information acquired by the pressure sensors ${ }^{[64]}$. Ji et al. employed the same ALL and put forward a new method named MUSIC (multiple signal classification) for the purpose of locating dipole source with high-resolution based on spatial spectrum estimation. Moreover, they also presented a MVDR (minimum variance distortionless response) method which improved the previous Capon's method (an adaptive beamforming-based method). For further study, MUSIC method showed a potential to locate two close dipole sources ${ }^{[76]}$. In 2019, Ji et al. established a quantitive and method-independent Cramer-Rao Lower Bound (CRLB) model to evaluate the localization performance of the above two methods, least square and MUSIC, which provided guidance on the optimal design of the ALL with the least sensors and the most appropriate spacing $^{[77]}$.

In 2018, Liu et al. developed an ALL consisting of 25 high precision pressure sensors (Fig. 11i). They established a mathematical model of the dipole source through Euler equation and plane potential flow theory to describe the relationship between the characteristic parameters of source and the surface pressure of the underwater vehicle and successfully detected the position, frequency and amplitude of the source through a neural network model. The consistency of simulation and experiment results demonstrated the effectiveness of the method ${ }^{[68]}$

In 2018, Yen and Guo adopted the potential flow theory to predict the hydrodynamic pressure and presented a method to follow periodic stimulus generated by an oscillating source. The fin of the robot was regarded as an oscillator. By subtracting the pressure induced by the robotic fish from the pressure measured by the PVDF sensor (Fig. 11h), they acquired the pressure 


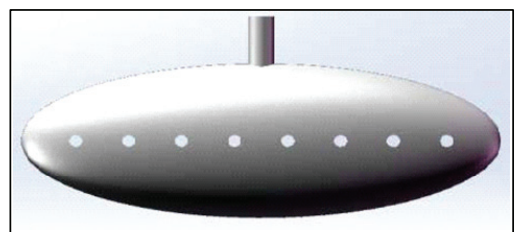

(a)

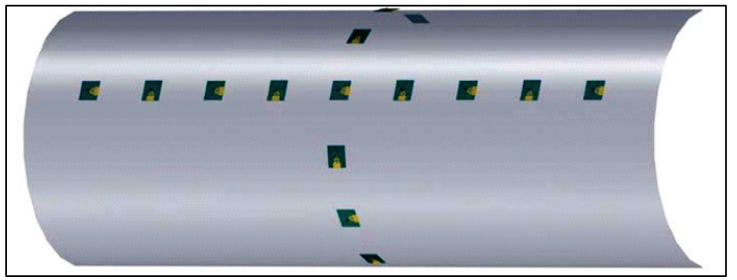

(c)

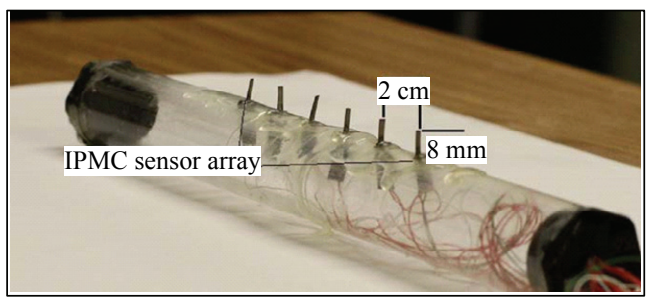

(f)

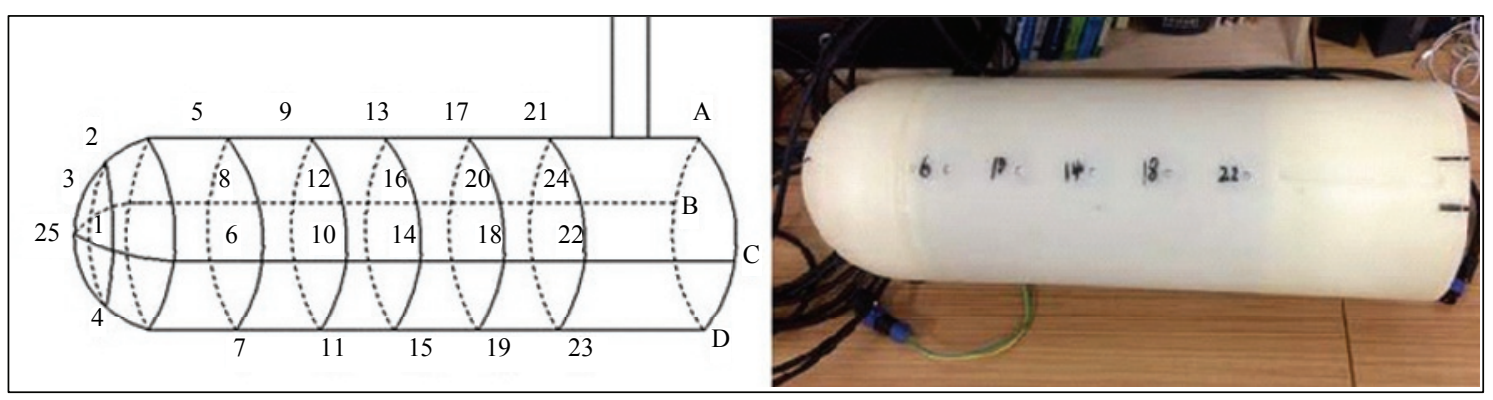

(i)

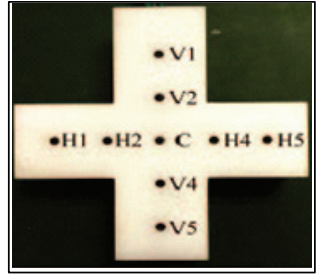

(d)

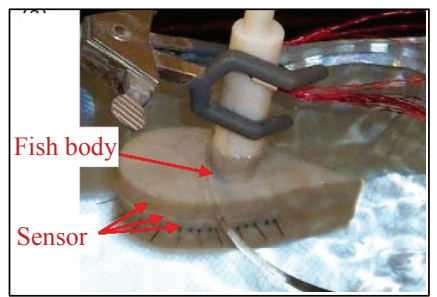

(g)

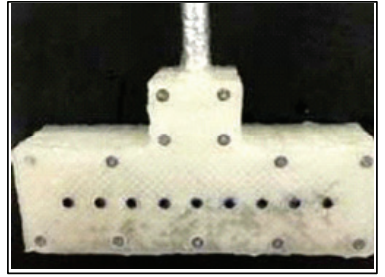

(e)

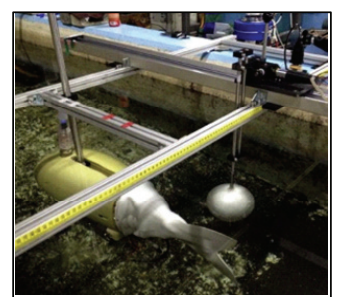

(h)

Fig. 11 Different carriers boarded with ALL mentioned in section 5. (a) The fish-shaped prototype inspired by the trout lateral line in Ref. [62]. (b) Photo of an AHC sensor array used in Ref. [30]. (c) Diagram of the ALL showing the biomimetic neuromast layout in Ref. [32]. (d) The design of ALL in Ref. [63]. (e) The design of ALL in Ref. [64]. (f) An experimental prototype of IPMC-based lateral line system used in Ref. [65]. (g) A prototype of an ALL with sensors used in Ref. [66]. (h) Robotic fish with the PVDF sensor along with the oscillating sphere in Ref. [67]. (i) Sensor layout and lateral line carrier physical map in Ref. [68].

generated by the source. Based on this, the robotic fish was able to adjust the amplitude, frequency, offset according to the phase difference ${ }^{[67]}$. Furthermore, this method lays a solid foundation for controlling the robotic fish to swim in a school.

In 2019, Wolf et al. used a 2D array of 8 all-optical sensors to measure the velocity profiles of a underwater object and then adopted feed-forward neural network and recurrent neural network to reconstruct the position of the object ${ }^{[78,79]}$. Furthermore, they imple- mented near field object classification based on hydrodynamic information with an Extreme Learning Machine neural network. This method provided more information about the shape compared to other 2D sensing array ${ }^{[80]}$.

In this section, we have put emphasis on discussing applications of ALL in locating and tracking the dipole source, especially the difference in approaches to reaching the results. Table 3 as follows can be a summary of this section. Flow induced by oscillating sources 
Table 3 Classification of existing studies in dipole source detection

\begin{tabular}{|c|c|c|}
\hline Author & ALL Sensors & Approaches \\
\hline Tang et al. $2019^{[62]}$ & 8 pressure sensors & $\begin{array}{l}\text { Linearizing the kinematic and dynamic conditions of the free } \\
\text { surface wave equation }\end{array}$ \\
\hline Yang et al. $2007^{[30]}$ & An array of $\mathrm{AHC}$ sensors & Extracting velocity amplitudes at the sphere vibration frequency \\
\hline Yang et al. $2010^{[32]}$ & 15 biomimetic neuromasts & Beamforming algorithm \\
\hline Pandya et al. $2006^{[44]}$ & $\begin{array}{c}\text { An array of } 16 \text { hot-wire anemo- } \\
\text { meters }\end{array}$ & Template training approach and the modeling approach \\
\hline Asadnia et al. $2013^{[33]}$ & An array of 2 by 5 pressure sensors & Measuring the maximum peak-to-peak out put of the sensors \\
\hline Abdulsadda et al. $2011^{[35]}$ & 5 IPMC sensors & Neural network \\
\hline Abdulsadda et al. $2013^{[65]}$, Chen et al. $2012^{[69,70]}$ & 6 IPMC sensors & Gauss Newton and Newton Raphson algorithms \\
\hline Ahrari et al. $2016^{[66]}$ & Multiple flow velocity sensors & Bi-level optimization methodology \\
\hline Zheng et al. $2018^{[63]}$ & $\begin{array}{l}9 \text { underwater pressure sensors } \\
\text { forming a cross }\end{array}$ & Generalized regression neural network \\
\hline Lin et al. $2018^{[64]}$ & 9 pressure sensors in a straight line & Least square method \\
\hline Ji et al. $2018^{[76]}$ & 9 pressure sensors in a straight line & MUSIC, MVDR \\
\hline Ji et al. $2019^{[77]}$ & 9 pressure sensors in a straight line & CRLB model \\
\hline Liu et al. $2018^{[68]}$ & 25 high precision pressure sensors & Euler equation, plane potential flow theory and neural network \\
\hline Yen et al. $2018^{[67]}$ & A PVDF sensor & Potential flow theory \\
\hline $\begin{array}{c}\text { Dagamseh et al. } 2009^{[71,72]} \text {, Dagamseh et al. } 2010^{[73,74]} \text {, } \\
\text { Dagamseh et al. } 2013^{[75]}\end{array}$ & An array of hair flow-sensors & $\begin{array}{c}\text { Detecting the parallel and the perpendicular velocity components, } \\
\text { beamforming techniques }\end{array}$ \\
\hline Wolf et al. $2019^{[78,79]}$, Wolf et al. 2020 $0^{[80]}$ & 8 all-optical flow sensors & Feed-forward neural network and recurrent neural network \\
\hline
\end{tabular}

is only one of the most basic forms of water flows and similar to wake flow generated by real fish. Based on the results above, we can also conduct natural environment experiments in which ALL is installed on robotic fish to follow real fish, even fish school and locate them in real time.

\section{Flow-aided control of underwater robots using ALL systems}

Lateral line plays an important part in sensing flow for fish school, which has inspired scientists worldwide to devote to developing underwater vehicles boarded with ALL systems consisting of arrays of sensors. The previous sections mainly presented applications based on a static ALL. If the ALL is moving with fish, the sensing difficulty will greatly increase. Assisted by ALL sensors, underwater vehicles can obtain fluid information accurately and effectively, providing a possibility to implement vehicles motion pattern identification and autonomous control. Varieties of experiments have been carried out in this domain, such as pattern identification, motion parameters (speed and direction) estimation and control, localization, obstacles detection and avoidance, energy consumption reduction and neighborhood ro- botic fish perception. Carriers boarded with ALL mentioned in this section are shown in Fig. 12.

In 2013, Akanyeti et al. firstly dived into the problem of hydrodynamic sensing on condition that the ALL is moving. Based on Bernoulli equation, they presented a formula about pressure detected by the ALL and the moving velocity and acceleration, which laid a solid foundation for the following study ${ }^{[87]}$. Additionally, Chambers et al. made a study of using a vertically or horizontally moving ALL to sense local flow. They came to a conclusion that a moving ALL performed better than static ${ }^{[88]}$. For further studies, a moving carrier boarded with ALL which has a great sensing ability can provide more valuable experimental data. The project FILOSE using a robotic fish showed in Fig. 10b not only aimed to study how fish perceive and respond to fluid simulation, but also constructed a bio-inspired robot on the basis of it. Boarded with ALL sensors, robotic fish measured the data from surrounding fluid environment which provided information on hydrodynamic features. And then by analysis, the relationship between fluid variables and kinestate of robotic fish was established, showing a new idea for robotic fish self-control. Several experiments have been 


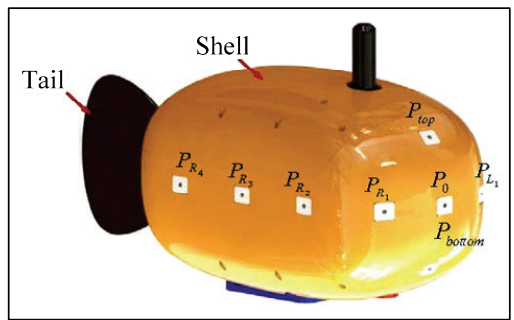

(a)

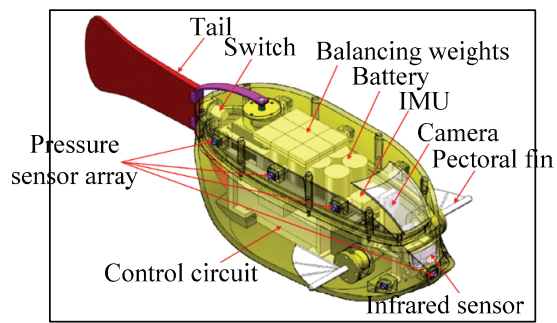

(b)

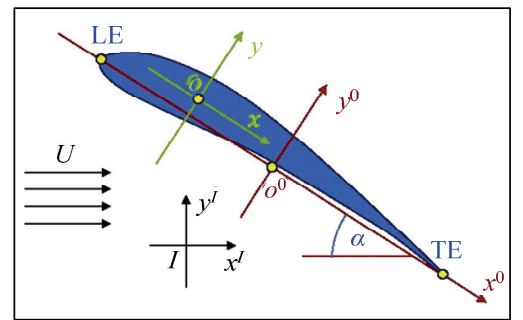

(c)
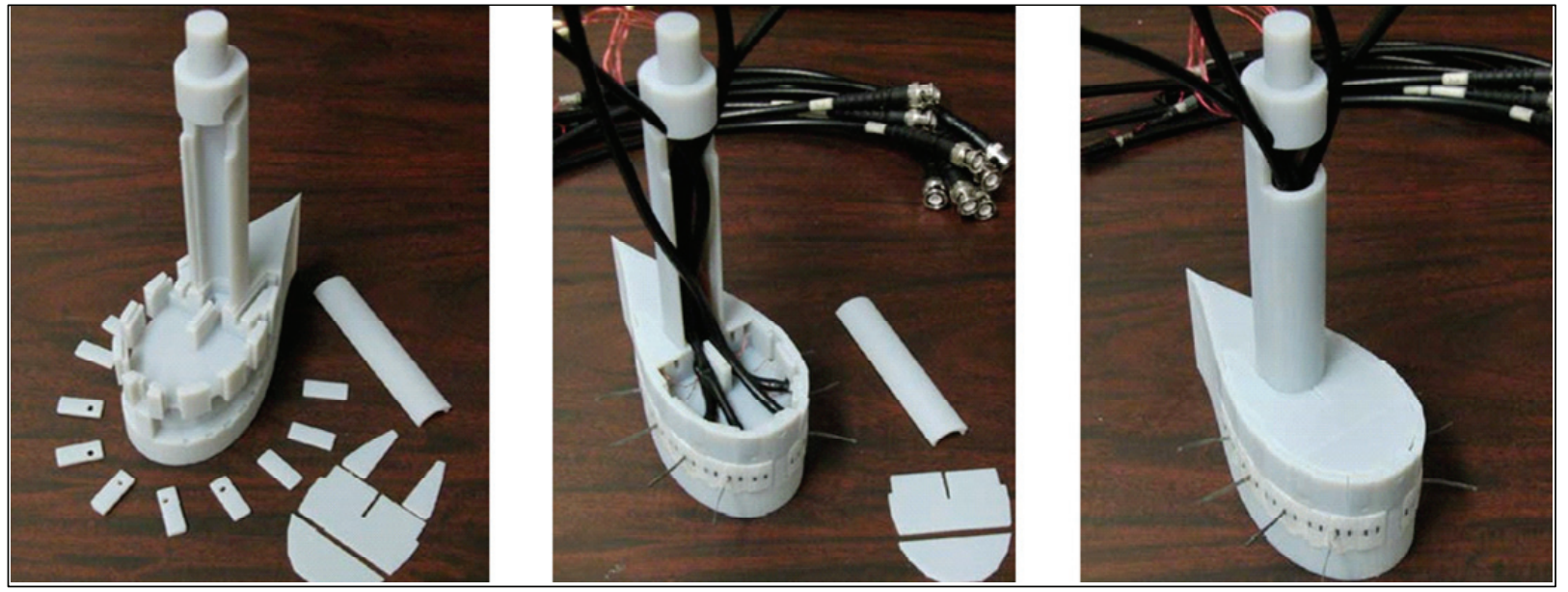

(d)

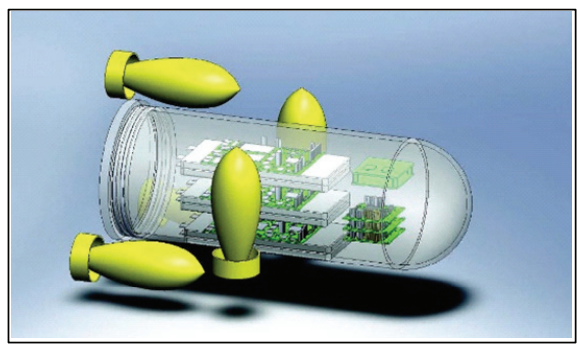

(e)

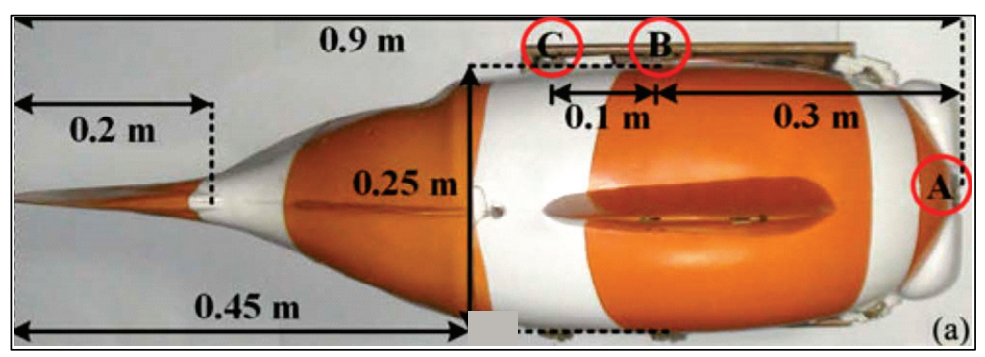

(f)

Fig. 12 Different carriers boarded with ALL mentioned in this section 6. (a) The robotic fish prototype in Ref. [81]. (b) The mechanical structure and electronics of the robotic fish used in Ref. [82]. (c) Illustration of reference frames I and O. TE denotes the trailing edge and LE the leading edge in Ref. [83]. (d) Modular design of robotic foil in Ref. [84]. An array of eight IPMC sensors is installed below an array of pressure sensors. (e) Conceptual 3D drawing of the vehicle in Ref. [85]. (f) Photographs of the robotic fish in Ref. [86].

conducted as follows: (1) detecting direction while swimming against the flow, (2) swimming along a predetermined trajectory, (3) maintaining stable position in constant current, (4) reducing energy consumption in turbulence, (5) reducing energy consumption by maintaining a stable position in the hydrodynamic shadow, (6) conducting control-experiments between real fish and robotic fish ${ }^{[89]}$. In this section, we will introduce flow-aided control of underwater robots using ALL systems by category.

\subsection{Pattern identification}

In 2014, Liu et al. conducted experiments that robotic fish (Fig. 12b) sense pressure information while swimming in different states such as forward swimming, turning, ascending and diving. And then based on feature points extracted from the data, they adopted a subtractive clustering algorithm to recognize the swimming states of robotic fish ${ }^{[90]}$. The success of this approach lays a solid foundation for quick control of robotic fish with 9 pressure sensors. 
In 2020, Zheng et al. made a breakthrough in motion parameters estimation of a robotic fish boarded with 11 pressure sensors (MS5803-01BA) (Fig. 12a). When the robotic fish moved at a specific state, such as rectilinear motion, turning motion, gliding motion, and spiral motion, they established a model combining the motion parameter including linear velocity, angular velocity, motion radius, etc. and the superficial hydrodynamic pressure variations. Robotic fish acquired the motion parameters based on the pressure detected by the ALL and then predicted the trajectory ${ }^{[91]}$. This work is of great importance for future study on self-trajectory-control.

\subsection{Motion parameters (speed and direction) esti- mation and control}

Kruusmaa et al. implemented rheotaxis behaviour in robotic fish in 2011. With the pressure sensors detecting flow information, they put forward a linear control law which helped the robotic fish to adjust the beat frequency in order to maintain position in the steady flow ${ }^{[92]}$. In 2013, they used a 50-cm-long robotic fish (Fig. 10b) mimicking the geometry and swimming mode of a rainbow trout and put forward a formula for estimating the speed. The experiments have proved the validity of it $^{[51]}$. Inspired by the Braitenberg vehicle $2 b$, they installed two pressure sensors on both sides of the head which could detect the pressure difference on the left and right sides of the robotic fish ${ }^{[93]}$. And then it was able to change the direction of swimming according to the difference to remain stable. Furthermore, they realized the position estimation and position stability of the robotic fish in steady water flows and behind solid objects $^{[51]}$.

Additionally, Wang et al. designed a robotic fish inspired by the geometry and swimming pattern of an ostraciiform boxfish shown in Fig. 12b. The robotic fish is boarded with an ALL composed of an array of 11 pressure sensors (Consensic CPS131) and an Inertial Measurement Unit (IMU). The former is used for fluid dynamic pressure data acquisition while the latter is used to monitor the robot pitch, yaw and roll angles. In order to reduce errors on account of sensors' inaccuracy and instability, they employed an optimal information fusion decentralized filter in 2015, as a consequence of which, the accuracy of speed estimation has been greatly im- proved. The speed estimation formula is derived from Bernoulli principle and corrected by local filter from ALL and IMU ${ }^{[82]}$. Furthermore, in 2016, they put forward a nonlinear prediction model including distributed pressure and angular velocity to estimate the speed of robotic fish ${ }^{[94]}$.

Moreover, Paley et al. also presented something new in flow speed and angle-of-attack detection in 2015. They used a new type of flexible robotic fish whose shape is a Joukowski-airfoil (Fig. 12c) with distributed pressure sensors. Flow speed and angle-of-attack were estimated by a recursive Bayesian filter assimilation pressure measurement. They combined an inverse-mapping feedforward controller based on an average model derived for periodic actuation of angle-of-attack and a proportional-integral feedback controller utilizing the estimated flow information to implement the closed-loop speed-control strategy ${ }^{[83,84,95]}$.

\subsection{Obstacles detection and avoidance}

Furthermore, extensive efforts have been done in obstacle detection and avoidance. Inspired by sensitivity of lateral line to the presence of oncoming currents and walls or obstacles, DeVries et al. developed a kind of wing underwater vehicle boarded with ALL sensors in 2015 (Fig. 12d). Employing potential flow theory, they simulated the flow field around vehicle in the situation that the flow was uniform and there were obstacles upstream. A nonlinear estimation model of free stream flow speed, attack of angle and relative position of obstacles by measuring local flow speed and pressure difference was derived theoretically. In order to implement the stability of swimming direction and position behind obstacles, they presented a recursive Bayesian filter. Finally, they discussed the Closed-loop control strate$\mathrm{gy}^{[84]}$.

In addition, Martiny et al. studied intensively in obstacles detection and avoidance in 2009. They developed an autonomous underwater vehicle equipped with 4 ALL sensors (Fig. 12e). Using hot-wire anemometry, the vehicle measured local flow speed around, which was proved related to the distance between obstacles and the vehicle theoretically and experimental$1 y^{[85]}$.

Yen et al. have made a breakthrough in obstacles 
detection and navigation in 2017. They used a robotic fish boarded with 3 ALL sensors (Fig. 12f) to measure nearby pressure variations, on the basis of which, they presented a way to control a robotic fish to swim along a straight wall. In theory, the tail of the robotic fish was regarded as an oscillating dipole in a 2D potential flow approximately and the wall effect was described by an image dipole on the opposite side of the wall. The robotic fish responded to the pressure variations in order to keep a fixed distance from the wall. A qualitative relationship between velocity and wall effect was concluded in this research ${ }^{[86]}$.

\subsection{Neighborhood robotic fish perception}

Not only have there been various results with respect to a single robotic fish, large amounts of experiments have also been carried out in multi-body control. Lots of work in this research field has been done by Wang et al. In 2015, they came to the conclusion by experiments that robotic fish (Fig. 12b) was capable of sensing the beating frequency of the robot swimming in front and the distance between the two robots with the help of ALL systems ${ }^{[96]}$. In 2017, they used the ALL system to detect the anti-Kármán-vortex-street-like vortex wake generated by its adjacent robotic fish. By extracting meaningful information from the pressure variations caused by the anti-Kármán-vortex- street-like vortex wake, the oscillating frequency, amplitude and offset of the adjacent robotic fish, the relative vertical distance and the relative yaw, pitch and roll angle between the robotic fish and its neighbor were sensed efficiently ${ }^{[97]}$. This progress lays a solid foundation for multi-body interactions research in the future.

In 2019, Zheng et al. used a robotic fish which is shown in Fig. 12a to conduct neighborhood perception experiments. Firstly, based on Bernoulli principles, they established a theoretical model to describe the hydrodynamic pressure variations on the surface of two adjacent robotic fish which swam diagonally ahead another ${ }^{[81]}$. Then, they utilized dye injection technique, hydrogen bubble technique and computational fluid dynamics simulation to study the vortices induced by the robotic fish separation. Besides, on the basis of the previous model, they also presented the relationship describing longitudinal separations and superficial hy- drodynamic pressure variations of two robotic fish ${ }^{[98]}$. This progress provided a new method for robotic fish school sensing.

In addition to the results mentioned above, some other applications of ALL in robotic fish control are introduced below. With respect to localization, $\mathrm{Mu}-$ hammad et al. produced preliminary results by flow feature extraction and comparison of compact flow features, based on which they developed an underwater landmark recognition technique in $2015^{[99]}$. This technique enables robotic fish to recognize locations that it has previously visited both in semi-natural and natural environments. In 2017, Fuentes-Pérez et al. proposed a map-based localization technique that employed simulated hydrodynamic maps. They used a computational fluid dynamics model to generate a flow rate diagram. Hydrodynamic information was acquired by ALL systems and analyzed to estimate the speed. Compared with the flow rate diagram, the location of the robotic fish was found out in the flow rate maps which was simulated from hydrodynamic results ${ }^{[100]}$.

As for energy consumption, Kruusmaa et al. conducted a control experiment that the robotic fish swims in steady flow, behind a cylinder and behind a cuboid in 2013. Consequently, swimming behind a cuboid consumed the least energy. They concluded that this phenomenon was on account of the presence of the well-defined suction zone behind the cylinder. Both obstacles avoidance and energy consumption reduction are of giant significance to the navigation of robotic fish ${ }^{[51]}$.

In this section, we have focused on the application of ALL systems in robotic fish control. Table 4 as follows lists different projects mentioned above and related ongoing studies. Similar to the previous sections, robotic fish in this section is mostly static or move in a simple state, such as rectilinear motion and turning motion in laboratory environment. However, the motion of real fish and the real underwater environment are much more complicated, which makes it more difficult for underwater sensing. To solve these problems, we need to improve the sensing system and establish new control algorithms for natural environment studies. Additionally, perception of underwater obstacles provides a new approach for underwater environment reconstruction and results of neighborhood robotic fish perception can be a 
Table 4 Classification of existing studies in flow-aided control

\begin{tabular}{|c|c|c|c|}
\hline Project & Author & ALL Sensors & $\begin{array}{c}\text { Laboratory experiment/ Natural } \\
\text { environment experiment }\end{array}$ \\
\hline \multirow{2}{*}{ Pattern identification } & Liu et al. $2014^{[91]}$ & 9 pressure sensors (CPS131) & Laboratory experiment \\
\hline & Zheng et al. $2020^{[90]}$ & 11 pressure sensors (MS5803-14BA) & Laboratory experiment \\
\hline \multirow{5}{*}{$\begin{array}{l}\text { Direction detection and } \\
\text { holding }\end{array}$} & $\begin{array}{l}\text { Salumäe et al. } 2012^{[93]} \text {, } \\
\text { Salumäe et al. } 2013^{[51]}\end{array}$ & 5 pressure sensors (Intersema MS5407-AM) & Laboratory experiment \\
\hline & Lagor et al. $2013^{[95]}$ & The sensors (MikroTip Catheter Pressure Transducers) & Laboratory experiment \\
\hline & Zhang et al. $2015^{[83]}$ & 6 pressure sensors (Servoflo MS5401-BM) & Laboratory experiment \\
\hline & DeVries et al. $2015^{[84]}$ & 8 IPMC sensors and four embedded pressure sensors & Laboratory experiment \\
\hline & Salumäe et al. $2013^{[51]}$ & 5 pressure sensors (Intersema MS5407-AM) & Laboratory experiment \\
\hline \multirow{4}{*}{ Speed estimation } & $\begin{array}{c}\text { Wang et al. } 2015^{[82]} \text {, Wang et } \\
\text { al. } 2016^{[94]}\end{array}$ & 11 pressure sensors (Consensic CPS131) & Laboratory experiment \\
\hline & Lagor et al. $2013^{[95]}$ & The sensors (MikroTip Catheter Pressure Transducers) & Laboratory experiment \\
\hline & Zhang et al. $2015^{[83]}$ & 6 pressure sensors (Servoflo MS5401-BM) & Laboratory experiment \\
\hline & DeVries et al. $2015^{[84]}$ & 8 IPMC sensors and four embedded pressure sensors & Laboratory experiment \\
\hline Position holding & Salumäe et al. $2013^{[51]}$ & 5 pressure sensors (Intersema MS5407-AM) & Laboratory experiment \\
\hline \multirow{2}{*}{ Localization } & Muhammad et al. $2015^{[99]}$ & 14 pressure sensors (Intersema MS5407-AM) & Natural environment experiment \\
\hline & Fuentes-Pérez et al. $2017^{[100]}$ & 16 pressure sensors & Natural environment experiment \\
\hline \multirow{3}{*}{$\begin{array}{l}\text { Obstacles detection and } \\
\text { avoidance }\end{array}$} & DeVries et al. $2015^{[84]}$ & 8 IPMC sensors and four embedded pressure sensors & Laboratory experiment \\
\hline & Martiny et al. $2009^{[85]}$ & 4 pressure sensors & Natural environment experiment \\
\hline & Yen et al. $2018^{[86]}$ & 3 pressure sensors (MS5803-01BA) & Laboratory experiment \\
\hline $\begin{array}{l}\text { Energy consumption reduc- } \\
\text { tion }\end{array}$ & Salumäe et al. $2014^{[51]}$ & 5 pressure sensors (Intersema MS5407-AM) & Laboratory experiment \\
\hline \multirow{2}{*}{$\begin{array}{l}\text { Neighboring robot fish } \\
\text { sensing }\end{array}$} & $\begin{array}{l}\text { Wang et al. } 2015^{[96]} \text {, Zheng et } \\
\text { al. } 2017^{[97]}\end{array}$ & 9 pressure sensors (MS5803-01BA) & Laboratory experiment \\
\hline & Zheng et al. $2019^{[81,98]}$ & 11 pressure sensors (MS5803-01BA) & Laboratory experiment \\
\hline
\end{tabular}

basis of control of multi robotic fish, both of which are potential to promote underwater exploration.

\section{Discussion}

In the previous sections, we have reviewed ALL sensors based on different principles and applications in flow field characteristics identification, dipole source detection and control of underwater robots. Although fish lateral line provides inspiration for the design of ALL sensors and underwater detection, it also serves as a strict standard for research. There have been great progress in this area, however, performance of existing ALL systems is still quite far from that of real fish.

ALL sensors which have been developed are no match for that of real fish through evolution in sensitivity, stability, coordination and information processing. We can optimize the design of the sensitive element and consider the resonance frequency for a better perception of ALL sensors. As for the stability, measurement errors in different temperature or pressure conditions should be taken into account. Additionally, waterproofing measures is necessary for the normal operation of sensors in the harsh conditions underwater. The development of new materials and micromachining technology provides possible methods for improvements in both areas. Not only should the performance of a single sensory unit be improved, the coordination of an array of ALL sensors is also important. Existing arrays of ALL sensors are mainly composed of a single type of sensors (pressure sensors or flow sensors) and arranged regularly, which is quite different from that of real fish. Real lateral line consists of SNs and CNs for a comprehensive perception of surrounding environment and sensing cells are distributed in a specific pattern for a better sensing. Using pressure sensors and flow sensors simultaneously is a potential method to optimize the array of ALL sensors. Besides, we can put forward evaluation indexes of ALL in order to find out the best placement of sensors on the surface of underwater robots. In terms of information processing, the fish lateral line has many different sensory functions, which can be a reference standard for ALL sensors. Many algorithms in velocity measurements and dipole source detection have been put forward, but the sensory ability of ALL does not stop here. ALL 
has the potential to sense the obstacles, fish schools and even reconstruct the surrounding water environment, which is a basis of follow-up research on control of underwater robots.

As for hydrodynamic characteristics identification, there have been many results based on ALL systems. However, existing results are mainly based on laboratory experiments where the motion of ALL carrier is simple such as static state and rectilinear motion and the water environment is stable. By contrast, the motion of real fish and underwater environment are much more complicated, which will greatly increase the difficulty of experiments. With the development of ALL sensors, natural experiments are necessary for complete imitation of real fish lateral line.

In dipole source detection, many methods and algorithms have been presented because oscillatory flow is one of the most basic flows and can be used to simulate the wake produced by the wagging tail of fish, which is important for further study on location and track of real fish. But the matching degree of oscillating flow and fish wake needs further exploration. On the basis of current results, more experiments on real-time location of fish school and mimicking the group behavior of fish may become a focus in the future.

Flow-aided control of underwater robots is a promising project which has a wide range of applications in marine exploration. The first priority is to establish the motion model of underwater robots based on hydrodynamics theory or date-driven methods which provide a way for self-identification of motion pattern in the conditions where it is impossible to observe. Establishment of models can be a theoretical instruction for control strategies. In addition, obstacles recognition and avoidance is another necessary ability for autonomous control. Based on the location of obstacles, robots are potential to reconstruct the surrounding environment and plan an optimal path for navigation. Except for a single robotic fish, perception and control of a robotic fish group may greatly improve the efficiency of underwater exploration and the success rate of underwater missions with the help of ALL.

\section{Conclusion}

In this article, we briefly introduced the morphol- ogy and mechanism of the lateral line for a further understanding. And then we put emphasis on discussing progress in biomimetics inspired by lateral line. Different kinds of sensors based on different principles have been explored, which provide a new approach for fluid information detection. Furthermore, scientists have arranged sensors to fabricate ALL systems on underwater vehicle bodies to assist in underwater detection, which is superior to traditional methods in applicability and flexibility. For hydrodynamic environment sensing and characteristic detection, we have made great progress in flow field characteristics identification, flow velocity and direction detection, vortex street properties detection. Additionally, various algorithms have been put forward to locate the dipole source and the ALL system has been used for the autonomous control of underwater robotic fish.

In future, based on existing results, we can devote efforts to different aspects to improve the performance of ALL systems. The development of ALL systems provides a powerful tool for ocean exploration. Although great efforts have been devoted in this area, there is a long way to go to realize the comparable sensory ability and autonomous control of robotic fish to real fish.

\section{Acknowledgment}

This work was supported in part by grants from the National Natural Science Foundation of China (NSFC, No. 91648120, 61633002, 51575005) and the Beijing Natural Science Foundation (No. 4192026).

Open Access This article is licensed under a Creative Commons Attribution 4.0 International License, which permits use, sharing, adaptation, distribution and reproduction in any medium or format, as long as you give appropriate credit to the original author(s) and the source, provide a link to the Creative Commons licence, and indicate if changes were made.

The images or other third party material in this article are included in the article's Creative Commons licence, unless indicated otherwise in a credit line to the material. If material is not included in the article's Creative Commons licence and your intended use is not permitted by statutory regulation or exceeds the permitted use, you will need to obtain permission directly 
from the copyright holder.

To view a copy of this licence, visit http://creativecommons.org/licenses/by/4.0/.

\section{References}

[1] Triantafyllou M S, Triantafyllou G S. An efficient swimming machine. Scientific American, 1995, 272, 64-70.

[2] Yu J Z, Liu L Z, Wang L, Tan M, Xu D. Turning control of a multilink biomimetic robotic fish. IEEE Transactions on Robotics, 2008, 24, 201-206.

[3] Liang J H, Wang T M, Wen L. Development of a two - joint robotic fish for real - world exploration. Journal of Field Robotics, 2011, 28, 70-79.

[4] Wang W, Xie G M. Online high-precision probabilistic localization of robotic fish using visual and inertial cues. IEEE Transactions on Industrial Electronics, 2014, 62, 1113-1124.

[5] Yu J Z, Wang M, Tan M, Zhang J W. Three-dimensional swimming. IEEE Robotics \& Automation Magazine, 2011, 18, 47-58.

[6] Crespi A, Lachat D, Pasquier A, Ijspeert A J. Controlling swimming and crawling in a fish robot using a central pattern generator. Autonomous Robots, 2008, 25, 3-13.

[7] Seo K, Chung S J, Slotine J J E. CPG-based control of a turtle-like underwater vehicle. Autonomous Robots, 2010, 28, 247-269.

[8] Ijspeert A J, Crespi A, Ryczko D, Cabelguen J M. From swimming to walking with a salamander robot driven by a spinal cord model. Science, 2007, 315, 1416-1420.

[9] Mogdans J, Bleckmann H. Coping with flow: Behavior, neurophysiology and modeling of the fish lateral line system. Biological Cybernetics, 2012, 106, 627-642.

[10] Northcutt R G. The phylogenetic distribution and innervation of craniate mechanoreceptive lateral lines. The $\mathrm{Me}$ chanosensory Lateral Line, Springer, New York, USA, 1989, 17-78.

[11] Maruska K P. Morphology of the mechanosensory lateral line system in elasmobranch fishes: Ecological and behavioral considerations. Environmental Biology of Fishes, 2001, 60, 47-75.

[12] Liu G J, Wang A, Wang X B, Liu P. A review of artificial lateral line in sensor fabrication and bionic applications for robot fish. Applied Bionics and Biomechanics, 2016, 5, $1-15$.

[13] Coombs S, Janssen J, Webb J F. Diversity of lateral line systems: Evolutionary and functional considerations. Sensory Biology of Aquatic Animals, Springer, New York, USA,
1988, 553-593.

[14] Münz H. Morphology and innervation of the lateral line system in Sarotherodon niloticus (L.)(cichlidae, teleostei). Zoomorphologie, 1979, 93, 73-86.

[15] Tan S Z. Underwater artificial lateral line flow sensors. Microsystem Technologies, 2014, 20, 2123-2136.

[16] Van Netten S M. Hydrodynamic detection by cupulae in a lateral line canal: Functional relations between physics and physiology. Biological Cybernetics, 2006, 94, 67-85.

[17] McHenry M J, Strother J A, Van Netten S M. Mechanical filtering by the boundary layer and fluid-structure interaction in the superficial neuromast of the fish lateral line system. Journal of Comparative Physiology A, 2008, 194, 795.

[18] Fan Z F, Chen J, Zou J, Bullen D, Liu C, Delcomyn F. Design and fabrication of artificial lateral line flow sensors. Journal of Micromechanics and Microengineering, 2002, 12, 655.

[19] Chen N N, Tucker C, Engel J M, Yang Y C, Pandya S, Liu C. Design and characterization of artificial haircell sensor for flow sensing with ultrahigh velocity and angular sensitivity. Journal of Microelectromechanical Systems, 2007, 16, 999-1014.

[20] McConney M E, Chen N N, Lu D, Hu H A, Coombs S, Liu C, Tsukruk V V. Biologically inspired design of hydrogel-capped hair sensors for enhanced underwater flow detection. Soft Matter, 2009, 5, 292-295.

[21] Qualtieri A, Rizzi F, Todaro M T, Passaseo A, Cingolani R, De Vittorio M. Stress-driven AIN cantilever-based flow sensor for fish lateral line system. Microelectronic Engineering, 2011, 88, 2376-2378.

[22] Qualtieri A, Rizzi F, Epifani G, Ernits A, Kruusmaa M, De Vittorio M. Parylene-coated bioinspired artificial hair cell for liquid flow sensing. Microelectronic Engineering, 2012, 98, 516-519.

[23] Kottapalli A G P, Asadnia M, Miao J M, Triantafyllou M. Touch at a distance sensing: Lateral-line inspired MEMS flow sensors. Bioinspiration \& Biomimetics, 2014, 9, 046011.

[24] Jiang Y G, Ma Z Q, Fu J C, Zhang D Y. Development of a flexible artificial lateral line canal system for hydrodynamic pressure detection. Sensors, 2017, 17, 1220.

[25] Kottapalli A G P, Bora M, Asadnia M, Miao J, Venkatraman S S, Triantafyllou M. Nanofibril scaffold assisted MEMS artificial hydrogel neuromasts for enhanced sensitivity flow sensing. Scientific Reports, 2016, 6, 19336.

[26] Fernandez V I, Hou S M, Hover F S, Lang J H, Triantafyllou M S. Lateral-Line Inspired MEMS-array Pressure Sensing 
for Passive Underwater Navigation. Massachusetts Institute of Technology, Sea Grant College Program, 2007.

[27] Yaul F M, Bulovic V, Lang J H. A flexible underwater pressure sensor array using a conductive elastomer strain gauge. Journal of Microelectromechanical Systems, 2012, 21, 897-907.

[28] Kottapalli A G P, Asadnia M, Miao J M, Barbastathis G, Triantafyllou M S. A flexible liquid crystal polymer MEMS pressure sensor array for fish-like underwater sensing. Smart Materials and Structures, 2012, 21, 115030.

[29] Chen J, Fan Z F, Zou J, Engel J, Liu C. Two-dimensional micromachined flow sensor array for fluid mechanics studies. Journal of Aerospace Engineering, 2003, 16, 85-97.

[30] Yang Y C, Chen N N, Tucker C, Engel J, Pandya S, Liu C. From artificial hair cell sensor to artificial lateral line system: development and application. Proceedings of IEEE 20th International Conference on Micro Electro Mechanical Systems (MEMS), Kobe, Japan, 2007, 577-580.

[31] Chen N N, Chen J, Engel J, Pandya S, Tucker C, Liu C. Development and characterization of high sensitivity bioinspired artificial haircell sensor. Proceedings of Solid-State Sensors, Actuators, and Microsystems Workshop, Hilton, Head, USA, 2006, 6, 4-8.

[32] Yang Y C, Nguyen N, Chen N N, Lockwood M, Tucker C, Hu H, Bleckmann H, Liu C, Jones D L. Artificial lateral line with biomimetic neuromasts to emulate fish sensing. Bioinspiration \& Biomimetics, 2010, 5, 016001.

[33] Asadnia M, Kottapalli A G P, Shen Z Y, Miao J M, Triantafyllou M. Flexible and surface-mountable piezoelectric sensor arrays for underwater sensing in marine vehicles. IEEE Sensors Journal, 2013, 13, 3918-3925.

[34] Asadnia M, Kottapalli A G P, Karavitaki K D, Warkiani M E, Miao J, Corey D P, Triantafyllou M. From biological cilia to artificial flow sensors: Biomimetic soft polymer nanosensors with high sensing performance. Scientific Reports, 2016, 6, 32955 .

[35] Abdulsadda A T, Tan X B. Underwater source localization using an IPMC-based artificial lateral line. IEEE International Conference on Robotics and Automation, Shanghai, China, 2011, 2719-2724.

[36] Asadnia M, Kottapalli A G P, Miao J, Warkiani M E, Triantafyllou M S. Artificial fish skin of self-powered micro-electromechanical systems hair cells for sensing hydrodynamic flow phenomena. Journal of the Royal Society Interface, 2015, 12, 20150322.

[37] Krijnen G, Lammerink T, Wiegerink R, Casas J. Cricket inspired flow-sensor arrays. IEEE Sensors Conference, At- lanta, USA, 2007, 539-546.

[38] Stocking J B, Eberhardt W C, Shakhsheer Y A, Calhoun B H, Paulus J R, Appleby M. A capacitance-based whisker-like artificial sensor for fluid motion sensing. IEEE Sensors Conference, Waikoloa, USA, 2010, 2224-2229.

[39] Van Baar J J, Dijkstra M, Wiegerink R J, Lammerink T S J, Krijnen G J M. Fabrication of arrays of artificial hairs for complex flow pattern recognition. IEEE Sensors Conference, Toronto, Canada, 2003, 332-336.

[40] Izadi N, Boer M J D, Berenschot J W, Krijnen G J M. Fabrication of superficial neuromast inspired capacitive flow sensors. Journal of Micromechanics and Microengineering, 2010, 20, 085041.

[41] Klein A, Bleckmann H. Determination of object position, vortex shedding frequency and flow velocity using artificial lateral line canals. Beilstein Journal of Nanotechnology, 2011, 2, 276-283.

[42] Große S, Schröder W. The micro-pillar shear-stress sensor MPS $^{3}$ for turbulent flow. Sensors, 2009, 9, 2222-2251.

[43] Wolf B J, Morton J A S, MacPherson W N, Van Netten S M. Bio-inspired all-optical artificial neuromast for 2D flow sensing. Bioinspiration \& Biomimetics, 2018, 13, 026013.

[44] Pandya S, Yang Y C, Jones D L, Engel J, Liu C. Multisensor processing algorithms for underwater dipole localization and tracking using MEMS artificial lateral-line sensors. EURASIP Journal on Advances in Signal Processing, 2006, 2006, 076593.

[45] Liu P, Zhu R, Que R Y. A flexible flow sensor system and its characteristics for fluid mechanics measurements. Sensors, 2009, 9, 9533-9543.

[46] Yang Y C, Chen J, Engel J, Pandya S, Chen N N, Tucker C, Coombs S, Jones D L, Liu C. Distant touch hydrodynamic imaging with an artificial lateral line. Proceedings of the National Academy of Sciences, 2006, 103, 18891-18895.

[47] Chen J, Engel J, Chen N, Pandya S, Coombs S, Liu C. Artificial lateral line and hydrodynamic object tracking. 19th IEEE International Conference on Micro Electro Mechanical Systems, Istanbul, Turkey, 2006, 694-697.

[48] Verma S, Papadimitriou C, Lüthen N, Arampatzis G, Koumoutsakos P. Optimal sensor placement for artificial swimmers. Journal of Fluid Mechanics, 2020, 884, A24.

[49] Xu D, Lv Z Y, Zeng H N, Bessaih H, Sun B. Sensor placement optimization in the artificial lateral line using optimal weight analysis combining feature distance and variance evaluation. ISA Transactions, 2019, 86, 110-121.

[50] Venturelli R, Akanyeti O, Visentin F, Ježov J, Chambers L D, Toming G, Brown J, Kruusmaa M, Megill W M, Fiorini P. 
Hydrodynamic pressure sensing with an artificial lateral line in steady and unsteady flows. Bioinspiration \& Biomimetics, 2012, 7, 036004.

[51] Salumäe T, Kruusmaa M. Flow-relative control of an underwater robot. Proceedings of the Royal Society $A$ : $M a-$ thematical, Physical and Engineering Sciences, 2013, 469, 20120671.

[52] Fuentes-Pérez J F, Tuhtan J A, Carbonell-Baeza R, Musall M, Toming G, Muhammad N, Kruusmaa M. Current velocity estimation using a lateral line probe. Ecological Engineering, 2015, 85, 296-300.

[53] Tuhtan J A, Fuentes-Perez J F, Toming G, Schneider M, Schwarzenberger $R$, Schletterer $M$, Kruusmaa $M$. Man-made flows from a fish's perspective: Autonomous classification of turbulent fishway flows with field data collected using an artificial lateral line. Bioinspiration \& Biomimetics, 2018, 13, 046006.

[54] Liu G J, Liu S K, Wang S R, Hao H H, Wang M M. Research on artificial lateral line perception of flow field based on pressure difference matrix. Journal of Bionic Engineering, 2019, 16, 1007-1018.

[55] Strokina N, Kämäräinen J K, Tuhtan J A, Fuentes-Pérez J F, Kruusmaa M. Joint estimation of bulk flow velocity and angle using a lateral line probe. IEEE Transactions on Instrumentation and Measurement, 2015, 65, 601-613.

[56] Tuhtan J A, Fuentes-Perez J F, Toming G, Kruusmaa M. Flow velocity estimation using a fish-shaped lateral line probe with product-moment correlation features and a neural network. Flow Measurement and Instrumentation, 2017, 54, $1-8$.

[57] Liu G J, Hao H H, Yang T T, Liu S K, Wang M M, Incecik A, Li Z X. Flow field perception of a moving carrier based on an artificial lateral line system. Sensors, 2020, 20, 1512.

[58] Ren Z, Mohseni K. A model of the lateral line of fish for vortex sensing. Bioinspiration \& Biomimetics, 2012, 7, 036016

[59] Free B, Patnaik M K, Paley D A. Observability-based path-planning and flow-relative control of a bioinspired sensor array in a Karman vortex street. American Control Conference (ACC), Seattle, USA, 2017, 548-554.

[60] Free B A, Paley D A. Model-based observer and feedback control design for a rigid Joukowski foil in a Kármán vortex street. Bioinspiration \& Biomimetics, 2018, 13, 035001.

[61] Bleckmann H. Reception of Hydrodynamic Stimuli in Aquatic and Semiaquatic Animals, Springer, New York, USA, 1994.

[62] Tang Z J, Wang Z, Lu J Q, Ma G Q, Zhang P F. Underwater robot detection system based on fish's lateral line. Electronics, 2019, 8, 566.

[63] Zheng X D, Zhang Y, Ji M J, Liu Y, Lin X, Qiu J, Liu G J. Underwater positioning based on an artificial lateral line and a generalized regression neural network. Journal of Bionic Engineering, 2018, 15, 883-893.

[64] Lin X, Zhang Y, Ji M J, Zheng X D, Lv K H, Qiu J, Liu G J. Dipole source localization based on least square method and 3D printing. IEEE International Conference on Mechatronics and Automation (ICMA), Changchun, China, 2018, 2203-2208.

[65] Abdulsadda A T, Tan X B. Nonlinear estimation-based dipole source localization for artificial lateral line systems. Bioinspiration \& Biomimetics, 2013, 8, 026005.

[66] Ahrari A, Lei H, Sharif M A, Deb K, Tan X B. Design optimization of an artificial lateral line system incorporating flow and sensor uncertainties. Engineering Optimization, $2017,49,328-344$.

[67] Yen W K, Guo J. Phase controller for a robotic fish to follow an oscillating source. Ocean Engineering, 2018, 161, 77-87.

[68] Liu G J, Gao S X, Sarkodie-Gyan T, Li Z X. A novel biomimetic sensor system for vibration source perception of autonomous underwater vehicles based on artificial lateral lines. Measurement Science and Technology, 2018, 29, 125102.

[69] Chen X F, Zhu G M, Yang X J, Hung D L S, Tan X B. Model-based estimation of flow characteristics using an ionic polymer-metal composite beam. IEEE/ASME Transactions on Mechatronics, 2012, 18, 932-943.

[70] Abdulsadda A T, Tan X B. Localization of a moving dipole source underwater using an artificial lateral line. Bioinspiration, Biomimetics, and Bioreplication, San Diego, USA, 2012, 833909.

[71] Dagamseh A M K, Lammerink T S J, Bruinink C M, Wiegerink R J, Krijnen G J. Dipole source localisation using bio-mimetic flow-sensor arrays. Procedia Chemistry, 2009, 1, 891-894.

[72] Dagamseh A M K, Lammerink T S J, Wiegerink R J, Krijnen G J. A simulation study of the dipole source localisation applied on bio-mimetic flow-sensor linear array. 12th Annual Workshop on Semiconductor Advances for Future Electronics and Sensors (SAFE), Veldhoven, Netherlands, 2009, 534-537.

[73] Dagamseh A M K, Krijnen G J M. Map estimation of air-flow dipole source positions using array signal processing. Annual Workshop on Semiconductor Advances for Future Electronics and Sensors, SAFE 2010, Veldhoven, 
Netherlands, 2010.

[74] Dagamseh A M K, Lammerink T S J, Kolster M L, Bruinink C M, Wiegerink R J, Krijnen G J. Dipole-source localization using biomimetic flow-sensor arrays positioned as lateral-line system. Sensors and Actuators A: Physical, 2010, 162, 355-360.

[75] Dagamseh A, Wiegerink R, Lammerink T, Krijnen G J. Imaging dipole flow sources using an artificial lateral-line system made of biomimetic hair flow sensors. Journal of the Royal Society Interface, 2013, 10, 20130162.

[76] Ji M J, Zhang Y, Zheng X D, Lin X, Liu G J, Qiu J. Resolution improvement of dipole source localization for artificial lateral lines based on multiple signal classification. Bioinspiration \& Biomimetics, 2018, 14, 016016.

[77] Ji M J, Zhang Y, Zheng X D, Lin X, Liu G J, Qiu J. Performance evaluation and analysis for dipole source localization with lateral line sensor arrays. Measurement Science and Technology, 2019, 30, 115107.

[78] Wolf B J, Van Netten S M. Hydrodynamic imaging using an all-optical 2D artificial lateral line. IEEE Sensors Applications Symposium (SAS), Sophia Antipolis, France, 2019, $1-6$.

[79] Wolf B J, Warmelink S, Van Netten S M. Recurrent neural networks for hydrodynamic imaging using a 2D-sensitive artificial lateral line. Bioinspiration \& Biomimetics, 2019, 14, 055001 .

[80] Wolf B J, Pirih P, Kruusmaa M, Van Netten S M. Shape classification using hydrodynamic detection via a sparse large-scale 2D-sensitive artificial lateral line. IEEE Access, 2020, 8, 11393-11404.

[81] Zheng X W, Xiong M L, Xie G M. Data-driven modeling for superficial hydrodynamic pressure variations of two swimming robotic fish with leader-follower formation. IEEE International Conference on Systems, Man and Cybernetics (SMC), Bari, Italy, 2019, 4331-4336.

[82] Wang C C, Wang W, Xie G M. Speed estimation for robotic fish using onboard artificial lateral line and inertial measurement unit. IEEE International Conference on Robotics and Biomimetics (ROBIO), Zhuhai, China, 2015, 285-290.

[83] Zhang F, Lagor F D, Yeo D, Washington P, Paley D A. Distributed flow sensing for closed-loop speed control of a flexible fish robot. Bioinspiration \& Biomimetics, 2015, 10, 065001 .

[84] DeVries L, Lagor F D, Lei H, Tan X, Paley D A. Distributed flow estimation and closed-loop control of an underwater vehicle with a multi-modal artificial lateral line. Bioinspiration \& Biomimetics, 2015, 10, 025002.
[85] Martiny N, Sosnowski S, Kühnlenz K, Hirche S, Nie Y, Franosch J M P, Van Hemmen J L. Design of a lateral-line sensor for an autonomous underwater vehicle. IFAC Proceedings Volumes, 2009, 42, 292-297.

[86] Yen W K, Sierra D M, Guo J W. Controlling a robotic fish to swim along a wall using hydrodynamic pressure feedback. IEEE Journal of Oceanic Engineering, 2018, 43, 369-380.

[87] Akanyeti O, Chambers L D, Ježov J, Brown J, Venturelli R, Kruusmaa M, Megill W M, Fiorini P. Self-motion effects on hydrodynamic pressure sensing: Part I. Forward-backward motion. Bioinspiration \& Biomimetics, 2013, 8, 026001.

[88] Chambers L D, Akanyeti O, Venturelli R, Ježov J, Brown J, Kruusmaa M, Fiorini P, Megill W M. A fish perspective: Detecting flow features while moving using an artificial lateral line in steady and unsteady flow. Journal of the Royal Society Interface, 2014, 11, 20140467.

[89] Kruusmaa M, Fiorini P, Megill W, de Vittorio M, Akanyeti O, Visentin F, Chambers L, Daou H E, Fiazza M C, Jezov J, Listak M, Rossi L, Salumae T, Toming G, Venturelli R, Jung D S, Brown J, Rizzi F, Qualtieri A, Maud J L. Filose for svenning: A flow sensing bioinspired robot. IEEE Robotics \& Automation Magazine, 2014, 21, 51-62.

[90] Liu H, Zhong K, Fu Y, Xie G, Zhu Q. Pattern recognition for robotic fish swimming gaits based on artificial lateral line system and subtractive clustering algorithms. Sensors \& Transducers, 2014, 182, 207.

[91] Zheng X W, Wang W, Xiong M L, Xie G M. Online state estimation of a fin-actuated underwater robot using artificial lateral line system. IEEE Transactions on Robotics, 2020, 36, 472-487.

[92] Kruusmaa M, Toming G, Salumäe T, Ježov J, Ernits A. Swimming speed control and on-board flow sensing of an artificial trout. IEEE International Conference on Robotics and Automation, Zhuhai, China, 2011, 1791-1796.

[93] Salumäe T, Ranó I, Akanyeti O, Kruusmaa M. Against the flow: A Braitenberg controller for a fish robot. IEEE International Conference on Robotics and Automation, Saint Paul, USA, 2012, 4210-4215.

[94] Wang W, Li Y, Zhang X, Wang C, Chen S, Xie G. Speed evaluation of a freely swimming robotic fish with an artificial lateral line. IEEE International Conference on Robotics and Automation (ICRA), Stockholm, Sweden, 2016, 4737-4742.

[95] Lagor F D, DeVries L D, Waychoff K M, Paley D A. Bio-inspired flow sensing and control: Autonomous underwater navigation using distributed pressure measurements. International Symposium on Unmanned Untethered 
Submersible Technology, Portsmouth, USA, 2013, 1-8.

[96] Wang W, Zhang X X, Zhao J W, Xie G M. Sensing the neighboring robot by the artificial lateral line of a bio-inspired robotic fish. IEEE/RSJ International Conference on Intelligent Robots and Systems (IROS), Hamburg, Germany, 2015, 1565-1570.

[97] Zheng X W, Wang C, Fan R F, Xie G M. Artificial lateral line based local sensing between two adjacent robotic fish. Bioinspiration \& Biomimetics, 2017, 13, 016002.

[98] Zheng X W, Wang M Y, Zheng J Z, Tian R Y, Xiong M L, Xie G M. Artificial lateral line based longitudinal separation sensing for two swimming robotic fish with leader-follower formation. IEEE/RSJ International Conference on Intelli- gent Robots and Systems (IROS), Macao, China, 2019, 2539-2544.

[99] Muhammad N, Strokina N, Toming G, Tuhtan J, Kruusmaa M. Flow feature extraction for underwater robot localization: Preliminary results. IEEE International Conference on Robotics and Automation (ICRA), Seattle, USA, 2015, $1125-1130$.

[100]Fuentes-Pérez J F, Muhammad N, Tuhtan J A, Carbonell-Baeza R, Kruusmaa M. Map-based localization in structured underwater environment using simulated hydrodynamic maps and an artificial lateral line. IEEE International Conference on Robotics and Biomimetics (ROBIO), Macao, China, 2017, 128-134. 